Prepared for the U.S. Department of Energy

under Contract DE-AC05-76RL01830

\title{
Preliminary Economics for the Production of Pyrolysis Oil from Lignin in a Cellulosic Ethanol Biorefinery
}

SB Jones

Y Zhu

April 2009

\section{Pacific Northwest}

NATIONAL LABORATORY 


\title{
DISCLAIMER
}

This report was prepared as an account of work sponsored by an agency of the United States Government. Neither the United States Government nor any agency thereof, nor Battelle Memorial Institute, nor any of their employees, makes any warranty, express or implied, or assumes any legal liability or responsibility for the aceuracy, completeness, or usefulness of any information, apparatus, produet, or process diselosed, or represents that its use would not infringe privately owned rights. Reference herein to any specific commercial product, process, or service by trade name, trademark, manufacturer, or otherwise does not necessarily constitute or imply its endorsement, recommendation, or favoring by the United States Government or any agency thereof, or Battelle Memorial Institute. The views and opinions of authors expressed herein do not necessarily state or reflect those of the United States Government or any agency thereof.

\author{
PACIFIC NORTHWEST NATIONAL LABORATORY \\ operated by \\ BATTELLE \\ for the \\ UNITED STATES DEPARTMENT OF ENERGY \\ under Contract DE-AC05-76RL01830
}

Printed in the United States of America
Available to DOE and DOE contractors from the Office of Scientific and Technical Information,
P.O. Box 62, Oak Ridge, TN 37831-0062;
ph: (865) 576-8401
fax: $(865) 576-5728$
email: reports@adonis.osti.gov

\begin{abstract}
Available to the public from the National Technical Information Service, U.S. Department of Commerce, 5285 Port Royal Rd., Springfield, VA 22161 ph: (800) 553-6847 fax: (703) 605-6900

email: orders@ntis.fedworld.gov

online ordering: http://www.ntis.gov/ordering.htm
\end{abstract}

This document was printed on recycled paper.

$(9 / 2003)$ 


\section{Preliminary Economics for the Production of Pyrolysis Oil from Lignin in a Cellulosic Ethanol Biorefinery}

SB Jones

Y Zhu

April 2009

Prepared for

the U.S. Department of Energy

under Contract DE-AC05-76RL01830

Pacific Northwest National Laboratory

Richland, Washington 99352 


\begin{abstract}
Cellulosic ethanol biorefinery economics can be potentially improved by converting by-product lignin into high valued products. Cellulosic biomass is composed mainly of cellulose, hemicellulose and lignin. In a cellulosic ethanol biorefinery, cellulose and hemicellullose are converted to ethanol via fermentation. The raw lignin portion is the partially dewatered stream that is separated from the product ethanol and contains lignin, unconverted feed and other by-products. It can be burned as fuel for the plant or can be diverted into higher-value products. One such higher-valued product is pyrolysis oil, a fuel that can be further upgraded into motor gasoline fuels. While pyrolysis of pure lignin is not a good source of pyrolysis liquids, raw lignin containing unconverted feed and by-products may have potential as a feedstock. This report considers only the production of the pyrolysis oil and does not estimate the cost of upgrading that oil into synthetic crude oil or finished gasoline and diesel.

A techno-economic analysis for the production of pyrolysis oil from raw lignin was conducted. comparing two cellulosic ethanol fermentation based biorefineries. The base case is the NREL 2002 cellulosic ethanol design report case where 2000 MTPD of corn stover is fermented to ethanol (NREL 2002). In the base case, lignin is separated from the ethanol product, dewatered, and burned to produce steam and power. The alternate case considered in this report dries the lignin, and then uses fast pyrolysis to generate a bio-oil product. Steam and power are generated in this alternate case by burning some of the corn stover feed, rather than fermenting it. This reduces the annual ethanol production rate from 69 to 54 million gallons/year. Assuming a pyrolysis oil value similar to Btu-adjusted residual oil, the estimated ethanol selling price ranges from $\$ 1.40$ to $\$ 1.48$ (2007 \$) depending upon the yield of pyrolysis oil. This is considerably above the target minimum ethanol selling price of \$1.33 for the 2012 goal case process as reported in the 2007 State of Technology Model (NREL 2008). Hence, pyrolysis oil does not appear to be an economically attractive product in this scenario. Further research regarding fast pyrolysis of raw lignin from a cellulosic plant as an end product is not recommended. Other processes, such as highpressure liquefaction or wet gasification, and higher value products, such as gasoline and diesel from fast pyrolysis oil should be considered in future studies.
\end{abstract}




\section{Acknowledgment}

The authors acknowledge the DOE EERE Office of Biomass Programs for funding this work and the modeling work performed by the National Renewable Energy Laboratory (NREL), which is publicly available and serves as the basis for cellulose to ethanol portion of the models. 


\section{Contents}

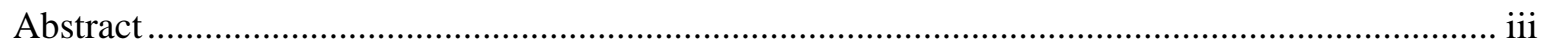

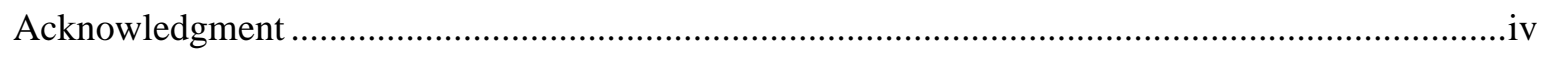

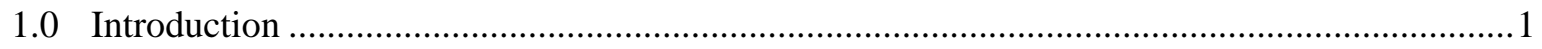

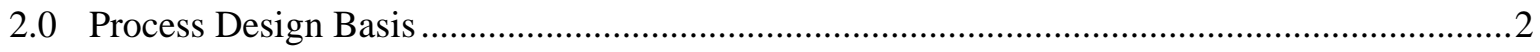

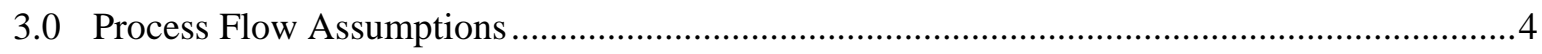

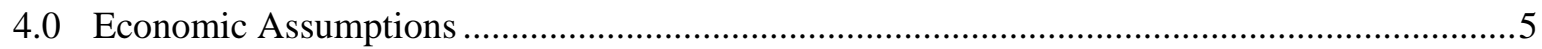

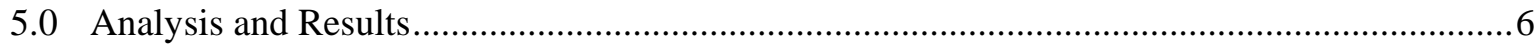

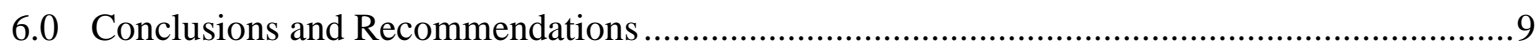

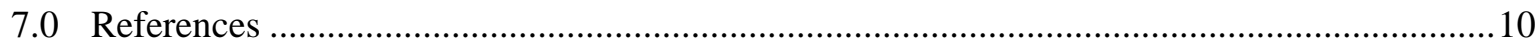

8.0 Appendix: Heat and Material Balance for the High Char Case.............................................11

\section{Figures}

Figure 1. Process Flowsheet for a Cellulosic Ethanol Plant Co-Producing Pyrolysis Oil .................2

Figure 2 Process Flowsheet for the Fast Pyrolysis System........................................................

Figure 3 Process Flowsheet for the High Char Case....................................................................12

\section{Tables}

Table 1. Process Assumption Summary for Fast Pyrolysis .........................................................

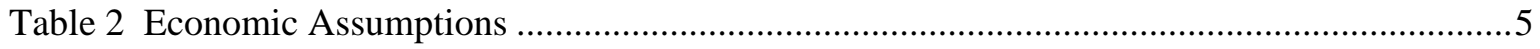

Table 3 Raw Material Consumption and By-Product Production ..................................................

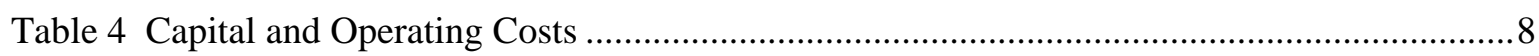

Table 5 Heat and Material Balance for the High Char Case ..........................................................16 


\subsection{Introduction}

The “Top Value-Added Chemicals from Biomass” report volumes I and II outline potential renewable fuels and chemicals from biomass (EERE 2004, PNNL 2007). This techno-economic analysis is a continuation of the detailed techno-economic analysis of specific products identified in these two volumes, as described in DOE project 4.5.1.2-10779. The objective of this task is to support the efforts of the Department of Energy's Office of Energy Efficiency and Renewable Energy, Office of the Biomass Program (EERE OBP) by conducting process and economic modeling of products from biomass sources. Specifically, this report analyzes the cost of producing pyrolysis oil from cellulosic-ethanol-derived raw lignin. Cellulosic ethanol biorefinery economics have the potential to be improved by converting byproduct lignin to make higher valued products than boiler fuel.

Cellulosic biomass is composed mainly of cellulose, hemicellulose and lignin. In a cellulosic ethanol plant, cellulose and hemicellullose are converted to ethanol via fermentation. The raw lignin portion is the partially dewatered stream containing lignin, unconverted feed and by-products. It can be burned as fuel for the plant or can be diverted into higher-value products. One such higher-valued product is pyrolysis oil, a fuel that can be further upgraded into motor gasoline fuels. While pyrolysis of pure lignin is not a good source of pyrolysis liquids, raw lignin containing unconverted feed and by-products may have potential as a feedstock. This report considers only the production of the pyrolysis oil and does not estimate the cost of upgrading that oil into synthetic crude oil or finished gasoline and diesel. 


\subsection{Process Design Basis}

The process flowsheet is based on the cellulosic-ethanol design case of the National Renewable Energy Laboratory (NREL 2002). A simplified block diagram of this process is shown in Figure 1.

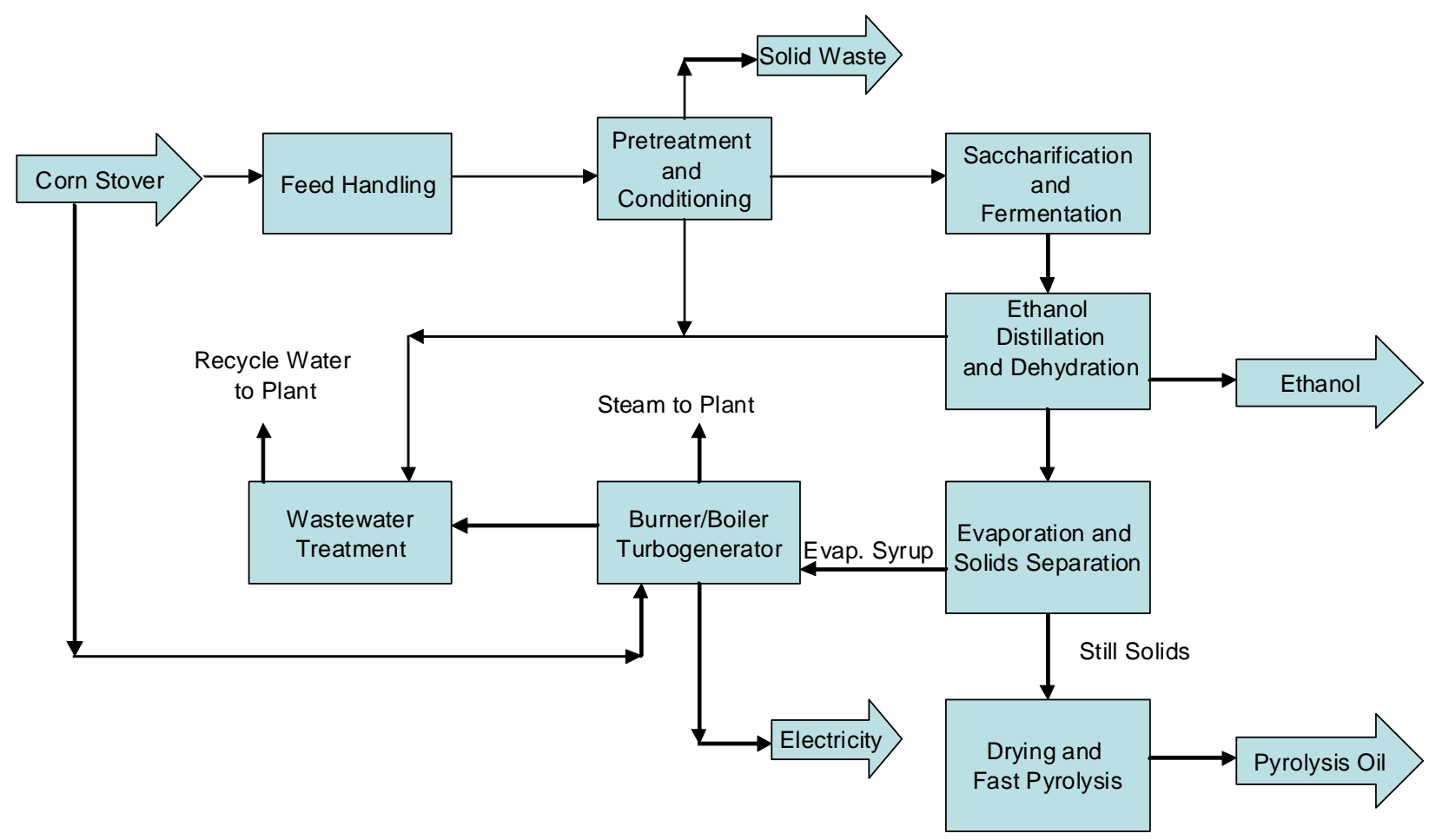

Figure 1. Process Flowsheet for a Cellulosic Ethanol Plant Co-Producing Pyrolysis Oil

Corn stover is washed and shredded. Pre-treatment with steam and dilute sulfuric acid releases the five- and six-carbon sugars in the hemicellulose. The pretreated feed is neutralized, and then sent to enzymatic saccharification to release the six-carbon sugars in the cellulose. This is followed by fermentation of the sugars to ethanol. The dilute ethanol stream is distilled to separate ethanol from the solids and the majority of the water. The ethanol is then dehydrated to $99 \%$ ethanol. The distillationbottoms stream is sent to a triple-effect evaporator to concentrate the solids. Two solid streams are generated: the mainly lignin portion ("still solids") from the first evaporator and the evaporator syrup from the third evaporator. In the base NREL process, both solid streams are sent to the boiler to raise steam for power generation. Also, in the NREL case, all power for the plant is generated onsite and any excess electricity is sold back to the grid. The details of this part of the process can be found in the NREL cellulosic ethanol design report (NREL 2002).

In the process discussed in this report, the lignin-rich "still solids" are sent to the fast-pyrolysis portion of the plant. The evaporator syrup supplies insufficient fuel to generate power for the entire plant; therefore, a portion of the corn stover feedstock is diverted to the boiler. The total feed rate of corn stover is maintained at 2000 dry mtpd. This size of plant is consistent with a delivered-feedstock cost of $\$ 46 /$ dry 
short ton (NREL 2008). At this price, the feedstock is assumed to "reactor-throat ready." That is, it has already been washed and size-reduced.

Figure 2 shows the equipment flow diagram for the fast-pyrolysis system. Wet lignin containing solids from the first evaporator are dried from $50 \%$ moisture to $8 \%$ moisture using hot exhaust gases. The fast- pyrolysis reactor system is assumed to be a circulating fluidized-bed reactor using hot sand to provide the heat for the endothermic pyrolysis reactions. The reactor residence time is less than 1 second. During this time, the lignin and other solids in the feed break down into non-condensable gases, water, char and organic vapor. The reactor effluent enters a cyclone to separate sand, char, and unconverted solids from the vapor product. The vapor product is then rapidly quenched with cooled pyrolysis oil product to prevent further reaction. A portion of the non-condensable gases is recycled to the pyrolysis reactor to help fluidize the bed. The remainder is burnt with the char to provide reheat to the circulating sand. Hot exhaust flue gases from the sand heater are sent to the dryer to dry the incoming wet feed.

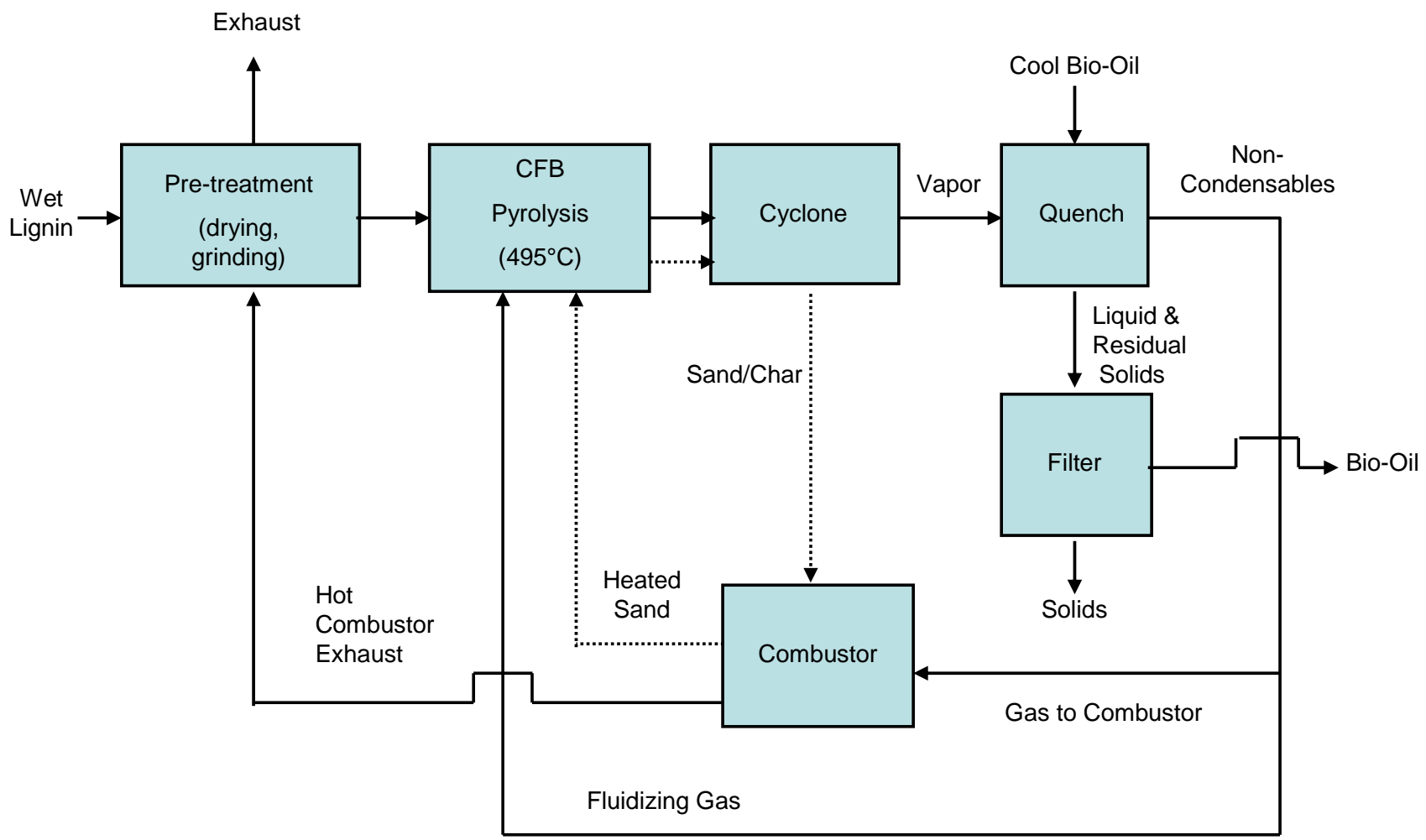

Figure 2 Process Flowsheet for the Fast Pyrolysis System

Three cases were modeled: the base case, the base pyrolysis case and a pyrolysis case with high char formation. The base case is the same as the state-of-technology case (NREL 2008). Here the plant produces only ethanol and by-product electricity. Since no experimental data exist for a lignin feed stream derived from a cellulosic ethanol plant, two cases pyrolysis cases were analyzed. One with high char yield and one with low char yield. In the high char case, the excess heat not needed by the dryer was recuperated into steam for use in the plant. 


\subsection{Process Flow Assumptions}

The flowsheet was modeled using the CHEMCAD process flow software. The simulation assumptions for all parts of the process except the fast-pyrolysis unit are those given in the NREL design report (NREL 2002). The fast-pyrolysis process is similar to that described in the PNNL Pyrolysis design report (PNNL 2008). Table 1 lists the assumptions and sources for the fast-pyrolysis reactor. No data were found for fast pyrolysis of raw lignin from a cellulosic ethanol plant. Thus the operating conditions were estimated from two extremes: sorghum bagasse containing approximately $25 \%$ lignin and milled wood lignin. The model feed on a dry basis is approximately $60 \%$ lignin with the remainder being ash and unconverted feed. Pyrolysis of whole lignocellulosic feedstocks is well documented and results in high conversions and yields as high as 75\% (wet) of pyrolysis oil (IEA 2007). At the other extreme is pure lignin. Pyrolysis of pure lignin feedstock is not easily done. Ferdous et. al. reports the pyrolysis of Alcell lignin at $520^{\circ} \mathrm{C}$ to have a $50 \%$ conversion with half the product being char and the remainder equally split between gas and liquid products (Ferdous 2001) .

Table 1. Process Assumption Summary for Fast Pyrolysis

\begin{tabular}{|c|c|c|c|c|c|}
\hline Process Area & $\begin{array}{l}\text { Process } \\
\text { Parameter }\end{array}$ & $\begin{array}{l}\text { Low Char } \\
\text { Case }\end{array}$ & $\begin{array}{l}\text { High Char } \\
\text { Case }\end{array}$ & $\begin{array}{l}\text { Literature } \\
\text { Values }\end{array}$ & Source \\
\hline \multirow[t]{4}{*}{ Lignin stream } & Water & $45 \%$ & $45 \%$ & & \\
\hline & Lignin & $33 \%$ & $33 \%$ & & \\
\hline & Ash & $10 \%$ & $10 \%$ & & \\
\hline & $\begin{array}{l}\text { Bio-solids and } \\
\text { by-products }\end{array}$ & $12 \%$ & $12 \%$ & & \\
\hline Pyrolysis feed dryer & $\begin{array}{l}\% \text { Moisture to } \\
\text { pyrolysis reactor }\end{array}$ & $8 \%$ & $8 \%$ & $8 \%$ & PNNL 2008 \\
\hline \multirow{5}{*}{$\begin{array}{l}\text { Fast pyrolysis } \\
\text { reactor }\end{array}$} & Temperature & $490^{\circ} \mathrm{C}$ & $499^{\circ} \mathrm{C}$ & $510^{\circ} \mathrm{C}$ & Piskorz $1998^{(\mathrm{a})}$ \\
\hline & Gas yield & $13 \%$ & $19 \%$ & $12.40 \%$ & Piskorz $1998^{(a)}$ \\
\hline & Char yield & $26 \%$ & $44 \%$ & $14.20 \%$ & Piskorz $1998^{(\mathrm{a})}$ \\
\hline & Water yield & $8 \%$ & $6 \%$ & $11.20 \%$ & Piskorz $1998^{(\mathrm{a})}$ \\
\hline & $\begin{array}{l}\text { Pyrolysis oil } \\
\text { yield }\end{array}$ & $53 \%$ & $31 \%$ & $62.20 \%$ & Piskorz $1998^{(\mathrm{a})}$ \\
\hline & C & $66 \%$ & $54 \%$ & $55 \%$ & Nunn $1985^{(b)}$ \\
\hline \multirow{2}{*}{ Wet basis } & $\mathrm{H}$ & $9 \%$ & $9 \%$ & $5 \%$ & Nunn $1985^{(b)}$ \\
\hline & $\mathrm{O}$ & $25 \%$ & $37 \%$ & $31 \%$ & Nunn $1985^{(b)}$ \\
\hline $\begin{array}{l}\text { Sorghu } \\
\text { milled }\end{array}$ & $\begin{array}{l}\text { bagasse } \\
\text { ood lignin }\end{array}$ & & & & \\
\hline
\end{tabular}




\subsection{Economic Assumptions}

The capital and operating costs are calculated in a Microsoft Excel spreadsheet using results from the CHEMCAD process simulation. A simple return-on-investment calculation is used to estimate the product selling price. All capital costs are reported in year-2007 dollars to be consistent with the current state of technology values (NREL 2008). The total capital investment is factored from installed equipment costs (NREL 2002). The operating labor was determined by assuming one operator per shift per major processing area. Most labor categories (control lab, supervisory, administrative) are factored from the operating labor. Maintenance materials, labor and local taxes and insurance are factored from the capital investment. These data and their sources are summarized in Table 2.

Table 2 Economic Assumptions

\begin{tabular}{|c|c|c|c|}
\hline & Value & Units or Basis & Reference \\
\hline Stream factor & $96 \%$ & & NREL 2002 \\
\hline Construction & Overnight $^{(\mathrm{a})}$ & & \\
\hline Plant life & 20 & Years & NREL 2002 \\
\hline Depreciation & 20 & Years straight line & \\
\hline Rate of return on capital & $10 \%$ & & \\
\hline \multicolumn{4}{|l|}{ Labor } \\
\hline Operating labor & 42 & $\begin{array}{l}\text { \$/hr Burdened with } 10 \% \text { shift } \\
\text { overlap }\end{array}$ & SRI PEP 2007 \\
\hline Maintenance labor & $1.0 \%$ & Of total installed capital (TIC) & SRI PEP 2007 \\
\hline Control lab labor & $20 \%$ & Of operating labor & SRI PEP 2007 \\
\hline Operator per shift per major unit & 1 & & \\
\hline \multicolumn{4}{|l|}{ Materials } \\
\hline Maintenance & $1.0 \%$ & Of TIC & SRI PEP 2007 \\
\hline Operating supplies & $10 \%$ & Of operating labor & SRI PEP 2007 \\
\hline Overhead & $80 \%$ & Of total labor & SRI PEP 2007 \\
\hline Local taxes and insurance & $2 \%$ & Of total fixed capital & SRI PEP 2007 \\
\hline General and administrative & $5 \%$ & Of product value & SRI PEP 2007 \\
\hline
\end{tabular}

(a) "Overnight construction" means no costs are associated with the construction phase, that is, construction is as if it happens "overnight." 


\subsection{Analysis and Results}

Table 3 summarizes the raw material consumption and by-product electricity generation for each case. Three cases are considered:

- $\quad$ Base Case: the lignin is burned in the boiler to produce plant steam and power,

- Fast Pyrolysis Low Char Case: the lignin is dried and then fast pyrolyzed with a minimum of char formation, some corn stover is diverted from fermentation for use as boiler feed, and

- Fast Pyrolysis High Char Case: the lignin is dried and then fast pyrolyzed with a maximum of char formation and some corn stover is diverted from fermentation for use as boiler feed.

The high-char-formation case was modeled to account for uncertainty in the pyrolysis yields. No data are available specifically for fast-pyrolysis yields from cellulosic ethanol lignin. Thus a worst case was modeled where the process yields more char than oil.

Chemical consumption per gallon of ethanol produced as shown in Table 3 is nearly the same for each case. However, the costs associated with each item are increased in the high and low char cases due to the reduced ethanol yield as compared with the base case. The fast pyrolysis oil produced in the high and low char cases are taken as an operating credit. It is assumed that the pyrolysis oil has the same value as residual oil with the price adjusted proportionately to reflect the difference in heating values of the two oils. All three cases generate power slightly in excess of that needed by the plant. Credit is taken for the excess power under the assumption that it can be sold to the grid.

Table 4 summarizes the yields and capital and operating costs for each case. Note that each case assumes a feed rate of 2000 MTPD dry stover to the plant gate. In the base case, most of the cellulosic and hemicellulosic material is fermented to ethanol. The lignin and any unconverted sugars are sent to the boiler to generate steam and power. When lignin is diverted from the boiler to a fast pyrolysis unit, another fuel must be substituted in the boiler. While additional stover above $2000 \mathrm{mtpd}$ could have been brought into the plant to serve as boiler fuel, the price will likely be higher than the \$46/ton assumed here. This is due to the additional transportation costs needed to widen the biomass collection area in order to increase the federate to the plant. Thus, in the fast pyrolysis scenario, a small portion of the corn stover was diverted from fermentation to the boiler. This reduces the available amount of cellulose and hemicellulose in the fermentation step, resulting in a $22 \%$ reduction in ethanol yield for the pyrolysis case. Routing part of the feed to the boiler also affects the capital costs. The capital cost of adding a pyrolysis unit is more than offset by the reduction in capital for the pretreatment, fermentation and product-separation portions of the plant. However, the value of pyrolysis oil is not sufficient to justify the reduction in ethanol yield, as can be seen by the estimated selling price.

It appears that producing pyrolysis oil from lignin will likely increase the selling price of ethanol by approximately 7-15 cents/gallon. 
Table 3 Raw Material Consumption and By-Product Production

\begin{tabular}{|c|c|c|c|c|c|c|c|c|c|c|c|}
\hline \multirow{3}{*}{ Raw Materials $^{(a)}$} & & & \multicolumn{3}{|c|}{$\begin{array}{c}\text { Base Case } \\
\text { No Pyrolysis Oil }\end{array}$} & \multicolumn{3}{|c|}{$\begin{array}{l}\text { Pyrolysis with Low } \\
\text { Char Formation Case }\end{array}$} & \multicolumn{3}{|c|}{$\begin{array}{l}\text { Pyrolysis with High } \\
\text { Char Formation Case }\end{array}$} \\
\hline & \multicolumn{2}{|c|}{ Unit Cost } & \multicolumn{2}{|c|}{$\begin{array}{l}\text { Consumption } \\
\text { /gal EtOH }\end{array}$} & \multirow[t]{2}{*}{ \$/gal } & \multicolumn{2}{|c|}{$\begin{array}{c}\text { Consumption } \\
\text { /gal EtOH }\end{array}$} & \multirow[t]{2}{*}{ \$/gal } & \multicolumn{2}{|c|}{$\begin{array}{l}\text { Consumption } \\
\text { /gal EtOH }\end{array}$} & \multirow[t]{2}{*}{ \$/gal } \\
\hline & & & & & & & & & & & \\
\hline Corn stover & 46 & $\begin{array}{l}\$ / \text { dry } \\
\text { ton }\end{array}$ & 0.011 & st & 51.20 & 0.014 & st & 66.11 & 0.014 & st & 66.11 \\
\hline Clarifier polymer & 1.299 & $\$ / l b$ & 0.000 & $\mathrm{lb}$ & 0.00 & 0.010 & $\mathrm{lb}$ & 1.23 & 0.010 & $\mathrm{lb}$ & 1.23 \\
\hline Sulfuric acid & 0.013 & $\$ / l b$ & 0.895 & $\mathrm{lb}$ & 1.16 & 0.895 & $\mathrm{lb}$ & 1.11 & 0.895 & $\mathrm{lb}$ & 1.11 \\
\hline Lime & 0.036 & $\$ / l b$ & 0.652 & $\mathrm{lb}$ & 2.36 & 0.652 & $\mathrm{lb}$ & 2.27 & 0.652 & $\mathrm{lb}$ & 2.27 \\
\hline Corn steep liquor & 0.084 & $\$ / l b$ & 0.351 & $\mathrm{lb}$ & 2.94 & 0.351 & $\mathrm{lb}$ & 2.83 & 0.351 & $\mathrm{lb}$ & 2.83 \\
\hline Purchased cellulase & 0.057 & $\$ / l b$ & 1.825 & $\mathrm{lb}$ & 10.48 & 1.825 & $\mathrm{lb}$ & 10.08 & 1.825 & $\mathrm{lb}$ & 10.08 \\
\hline $\begin{array}{l}\text { Diammonium } \\
\text { phosphate }\end{array}$ & 0.073 & $\$ / l b$ & 0.044 & $\mathrm{lb}$ & 0.32 & 0.044 & $\mathrm{lb}$ & 0.31 & 0.044 & $\mathrm{lb}$ & 0.31 \\
\hline Propane & 0.002 & $\$ / l b$ & 0.005 & $\mathrm{lb}$ & 0.00 & 0.005 & $\mathrm{lb}$ & 0.00 & 0.005 & $\mathrm{lb}$ & 0.00 \\
\hline Make-up water & 0.000 & $\$ / l b$ & 46.187 & $\mathrm{lb}$ & 0.56 & 46.187 & $\mathrm{lb}$ & 0.54 & 46.187 & $\mathrm{lb}$ & 0.54 \\
\hline BFW Chemicals & 1.403 & $\$ / l b$ & 0.000 & $\mathrm{lb}$ & 0.04 & 0.000 & $\mathrm{lb}$ & 0.04 & 0.000 & $\mathrm{lb}$ & 0.04 \\
\hline $\begin{array}{l}\text { Cooling water } \\
\text { chemicals }\end{array}$ & 1.061 & $\$ / l b$ & 0.001 & $\mathrm{lb}$ & 0.06 & 0.001 & $\mathrm{lb}$ & 0.06 & 0.001 & $\mathrm{lb}$ & 0.06 \\
\hline WWT Chemicals & 0.164 & $\$ / l b$ & 0.013 & $\mathrm{lb}$ & 0.22 & 0.013 & $\mathrm{lb}$ & 0.21 & 0.013 & $\mathrm{lb}$ & 0.21 \\
\hline WWT Polymer & 2.652 & $\$ / l b$ & 0.000 & $\mathrm{lb}$ & 0.01 & 0.000 & $\mathrm{lb}$ & 0.01 & 0.000 & $\mathrm{lb}$ & 0.01 \\
\hline Ash disposal & 0.009 & $\$ / l b$ & 1.214 & $\mathrm{lb}$ & 1.14 & 1.214 & $\mathrm{lb}$ & 1.14 & 1.214 & $\mathrm{lb}$ & 1.14 \\
\hline Gypsum disposal & 0.009 & $\$ / l b$ & 1.966 & $\mathrm{lb}$ & 1.84 & 1.966 & $\mathrm{lb}$ & 1.84 & 1.966 & $\mathrm{lb}$ & 1.84 \\
\hline $\begin{array}{l}\text { Gross Raw Material } \\
\text { Cost }\end{array}$ & & & & & 72.32 & & & 87.76 & & & 87.76 \\
\hline \multicolumn{12}{|l|}{$\begin{array}{l}\text { By-product } \\
\text { credits: }^{(b)}\end{array}$} \\
\hline Pyrolysis oil & 120 & $\$ /$ gal & 0.00 & gal & 0.00 & -0.20 & gal & -29.22 & -0.08 & gal & -20.19 \\
\hline \multicolumn{12}{|l|}{ Utilities } \\
\hline Electricity $^{(\mathrm{c})}$ & 4 & $\$ / \mathrm{kwh}$ & -2.12 & kwh & -8.49 & -1.48 & kwh & -5.20 & -2.54 & kwh & -6.41 \\
\hline $\begin{array}{l}\text { (a) Unit costs from } \mathrm{N} \\
\text { (b) Pyrolysis oil valu } \\
\text { (c) Electricity costs } \mathrm{f}\end{array}$ & design & se esca & $\begin{array}{l}\text { to } 2007 \\
\text { ice for } \mathrm{r} \\
\text { alated to }\end{array}$ & $\begin{array}{l}\text { ith th } \\
\text { idual } \\
007\end{array}$ & esign-c & $\begin{array}{l}\text { se work } \\
\text { sted fo } \\
\text { gn-case }\end{array}$ & heet ( & $\begin{array}{l}\text { EL } 20 \\
\text { tent. } \\
\text { t (NRI }\end{array}$ & 2002). & & \\
\hline
\end{tabular}

The capital and operating costs are shown in Table 4. 
Table 4 Capital and Operating Costs

\begin{tabular}{|c|c|c|c|}
\hline & & $\begin{array}{l}\text { Low Char } \\
\text { Formation }\end{array}$ & $\begin{array}{l}\text { High Char } \\
\text { Formation }\end{array}$ \\
\hline & Base Case & Case & \\
\hline Ethanol, million gal/yr & 69 & 54 & 54 \\
\hline MTPD dry stover & 2000 & 2000 & 2000 \\
\hline \multicolumn{4}{|l|}{ Investment, U.S. \$ million } \\
\hline Total installed capital & 135 & 127 & 127 \\
\hline Total indirect costs & 85 & 80 & 80 \\
\hline Total fixed capital & 220 & 207 & 207 \\
\hline \multicolumn{4}{|l|}{ Production costs, U.S. \$/gal } \\
\hline Raw materials & 0.72 & 0.88 & 0.88 \\
\hline By-products & 0.00 & -0.29 & -0.20 \\
\hline Utilities & -0.08 & -0.05 & -0.06 \\
\hline Variable costs, U.S. \$/gal & 0.64 & 0.53 & 0.61 \\
\hline Maintenance materials & 0.04 & 0.05 & 0.05 \\
\hline Operating supplies & 0.00 & 0.00 & 0.00 \\
\hline Operating labor & 0.03 & 0.04 & 0.04 \\
\hline Maintenance labor & 0.02 & 0.02 & 0.02 \\
\hline Control laboratory & 0.01 & 0.01 & 0.01 \\
\hline Total direct costs, U.S. \$/gal & 0.73 & 0.66 & 0.74 \\
\hline Plant overhead & 0.03 & 0.04 & 0.04 \\
\hline Taxes and insurance & 0.03 & 0.04 & 0.04 \\
\hline Depreciation & 0.16 & 0.19 & 0.19 \\
\hline \multirow[t]{2}{*}{ Plant gate cost, U.S. \$/gal } & 0.95 & 0.93 & 1.01 \\
\hline & 0.00 & 0.00 & 0.00 \\
\hline \multirow[t]{2}{*}{$\begin{array}{l}\text { General and administrative, sales } \\
\text { and research }\end{array}$} & 0.07 & 0.08 & 0.08 \\
\hline & & 0.00 & \\
\hline Production costs, U.S. \$/gal & 1.02 & 1.02 & 1.09 \\
\hline $\begin{array}{l}\text { Selling price, U.S. \$/gal (10\% } \\
\text { ROI) }\end{array}$ & 1.33 & 1.40 & 1.48 \\
\hline
\end{tabular}




\subsection{Conclusions and Recommendations}

A techno-economic analysis (TEA) using a CHEMCAD-based process model and Excel-based production-cost spreadsheet of cellulosic-derived lignin conversion to pyrolysis oil was performed. Fast pyrolysis of raw lignin from a cellulosic plant has not been demonstrated. Furthermore, the minimum amount of cellulosic material needed in the lignin stream to allow high conversions and high yields of liquid products is not known. However, this screening study suggests that producing fast pyrolysis oil as an end product in a biorefinery is too low of a value fuel to positively impact ethanol economics. Thus further research into fast pyrolysis as an end product is not recommended. If a higher valued product could be produced, then this option may have merit. Examples of higher valued products are upgrading the fast pyrolysis oil to gasoline or extracting chemicals such as levoglucosan. Estimating the economics for these higher valued products is beyond the scope of this work. However, this work can serve as a basis for future biorefinery studies.

Upgrading the raw lignin stream from an ethanol biorefinery to valuable products has the potential to improve the economics of a cellulosic biorefinery. It is recommended that techno-economic work be continued to identify those products worth pursuing. Since the lignin has high moisture content, it is recommended that future studies consider wet processing methods such as high-pressure liquefaction or gasification for fuels production. Upgrading fast pyrolysis oil and chemical production should also be considered. 


\subsection{References}

EERE. 2004. Werpy T et al. 2004. Top Value Added Chemicals from Biomass Volume I: Results of Screening for Potential Candidates from Sugars and Synthesis Gas. DOE/GO-102004-1992, U.S. Department of Energy, Richland, Washington.

Ferdous, D. el.al. 2001. Fuel Processing Technology. 70:9-26.

IEA 2007. Biomass Pyrolysis. IEA Bioenergy. Task 34 Booklet. T34:2007:01

http://www.ieabioenergy.com/MediaItem.aspx?id=5416

NREL. 2002. Aden et al. 2002. Lignocellulosic Biomass to Ethanol Process Design and Economics Utilizing Co-Current Dilute Acid Prehydrolysis and Enzymatic Hydrolysis for Corn Stover. NREL/TP510-32438, National Renewable Energy Laboratory, City, State.

NREL. 2008. Aden A. 2008. Biochemical Production of Ethanol from Corn Stover: 2007 State of Technology Model. NREL/TP-43205, National Renewable Energy Laboratory, City, State.

Nunn T. et.al. 1985. Industrial Engineering and Chemical Process Design and Development. 24:844852.

Piskorz J. et al. 1998. Journal of Analytical and Applied Pyrolysis. 46:15-29.

PNNL. 2007. Holladay J. et al. 2007. Top Value Added Chemicals from Biomass Volume II: Results of Screening for Potential Candidates from Biorefinery Lignin. PNNL-16983, Pacific Northwest National Laboratory, Richland, Washington.

PNNL. 2008. Jones S.B. et al. 2008. Production of Gasoline and Diesel from Biomass via Fast Pyrolysis, Hydrotreating and Hydrocracking: A Design Case. In review. PNNL-18284, Pacific Northwest National Laboratory, Richland, Washington. 


\subsection{Appendix: Heat and Material Balance for the High Char Case}




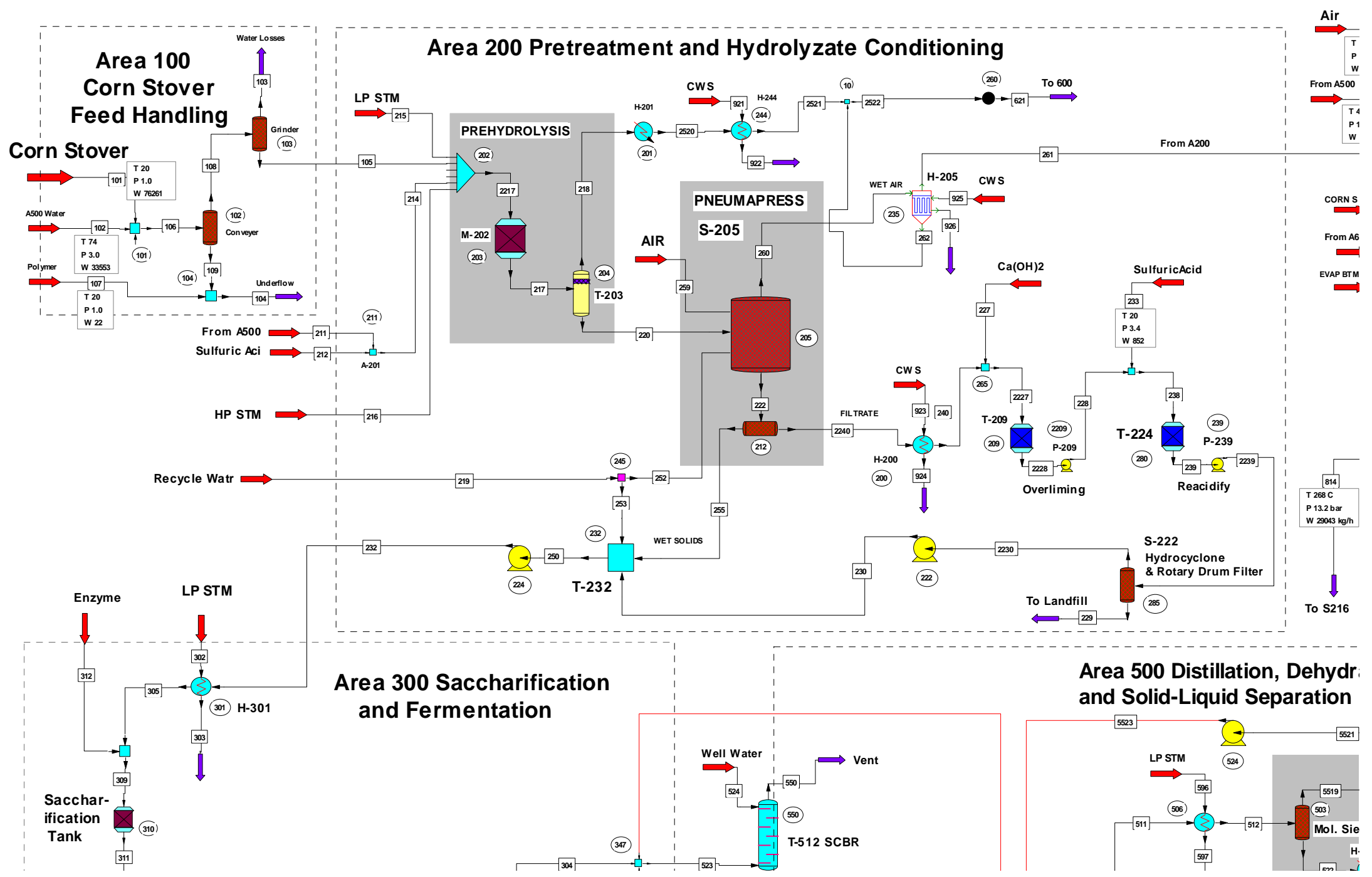

Figure 3 Process Flowsheet for the High Char Case 


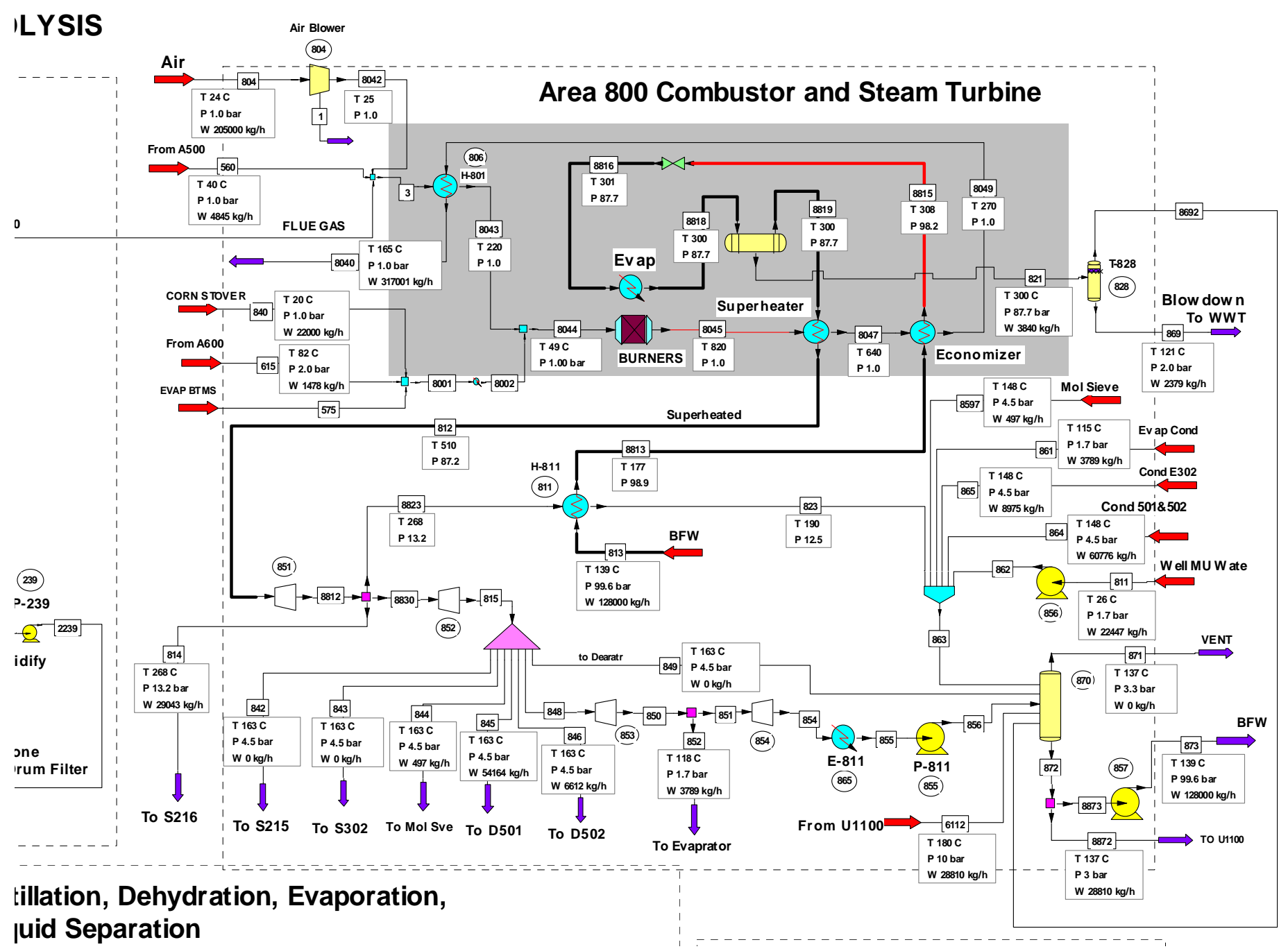

Figure 3, cont. Process Flowsheet for the High Char Case 


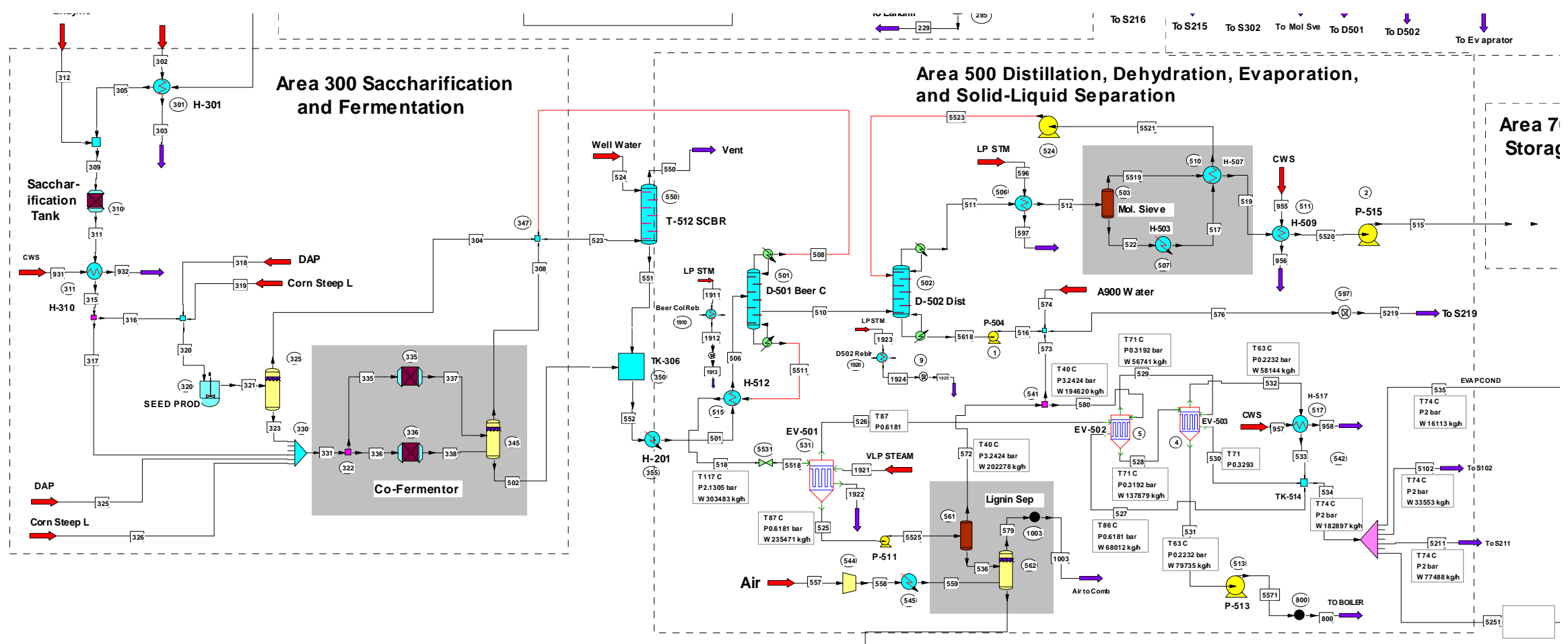

Figure 3, cont. Process Flowsheet for the High Char Case 


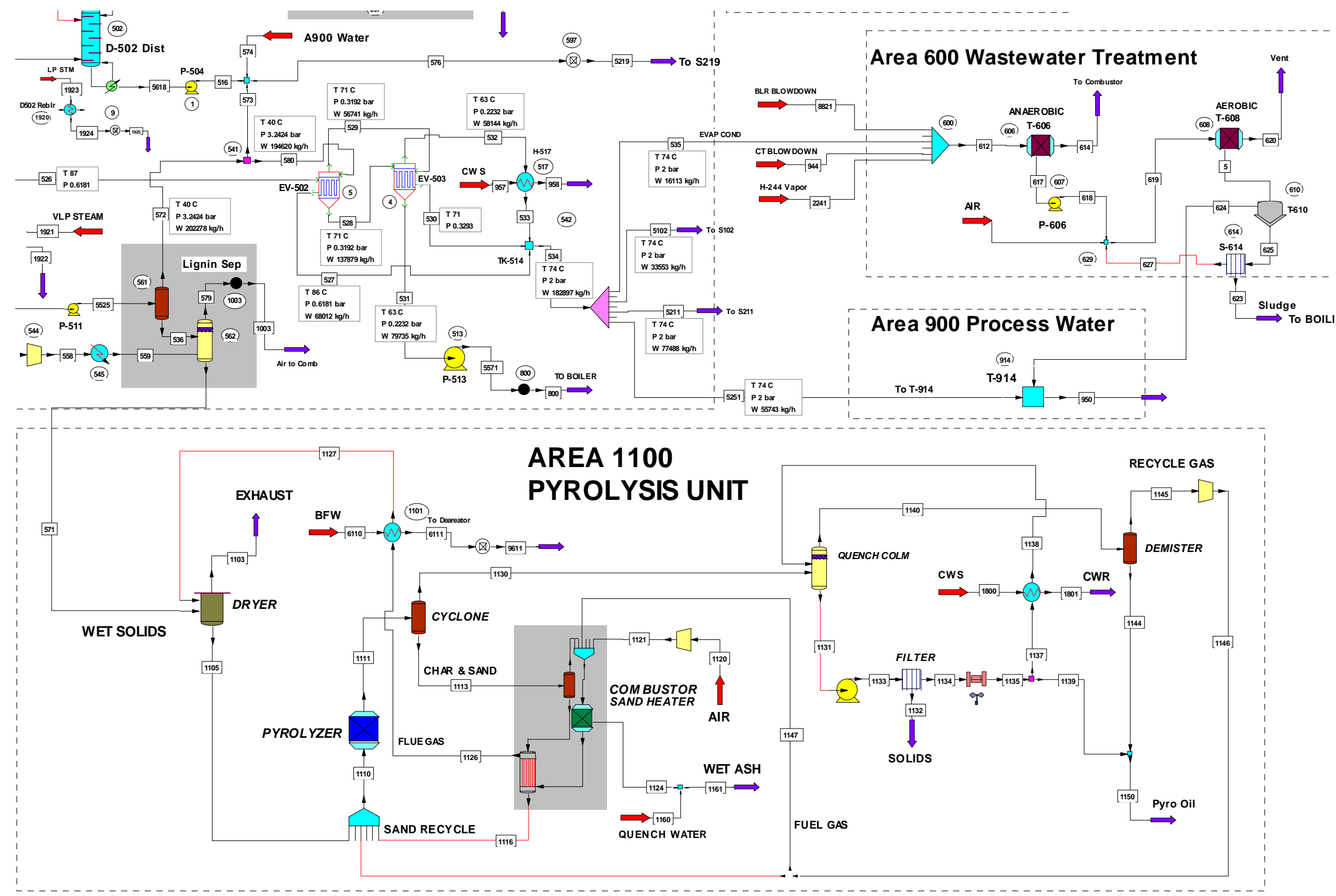

Figure 3, cont. Process Flowsheet for the High Char Case 
Table 5 Heat and Material Balance for the High Char Case

Stream No.
Stream Name
Temp C
Pres bar
Enth MW
Vapor mass fraction
Total kgh
Fowrates in kg/h
Glucose
Cellulose
Xylose
Xylan
Lignin
Acetate
Organism
Solslds
Gypsum
Lgnsol
HMF
C5Sugar
C6Sugar
C5Solid
C6Solid
CaH2O2
Ash
Ethanol
Water
Furfural
Sulfuric Acid
Nitrogen
Carbon Dioxide
Oxygen
Ammonia
Lactic Acid
Acetic Acid
Methane
Nitrogen Dioxide
Sulfur Dioxide
DAP
2-2-4-TriMth-C5
Hydrogen
Carbon
Carbon Monoxide

\begin{tabular}{|c|c|c|c|c|c|c|c|c|}
\hline \multirow[t]{2}{*}{1} & 3 & 5 & \multirow{2}{*}{$\begin{aligned} & 101 \\
& \text { Corn Stover }\end{aligned}$} & 102 & 103 & 104 & \multirow{2}{*}{\multicolumn{2}{|c|}{ Underflow }} \\
\hline & & & & A500 Water & \multicolumn{2}{|c|}{ Underflow } & & \\
\hline 25.0489 & 25.6756 & 21 & 20 & 73.8 & 45 & -26.44 & 45 & -27.7742 \\
\hline 1.0234 & 1 & 0.9818 & 1.0132 & 3.0397 & 1.0132 & 1.0132 & 1.0132 & 1.0132 \\
\hline 0 & -1.4844 & -309.27 & -163.13 & -144.29 & -38.56 & -3.4701 & -260.41 & -307.41 \\
\hline 0 & 1 & 0 & 0 & 0 & 0 & 0 & 0 & \\
\hline 0 & 213776.27 & 70051.4874 & 76261 & 33553 & 8912.7038 & 786.3577 & 100137.043 & 109814 \\
\hline 0 & 0 & 0 & 0 & 0 & 0 & 0 & 0 & \\
\hline 0 & 0 & 0 & 24261.5609 & 0 & 0 & 0 & 24261.5609 & 24261.5609 \\
\hline 0 & 0 & 0 & 0 & 0 & 0 & 0 & 0 & \\
\hline 0 & 0 & 0 & 13666.3191 & 0 & 0 & 0 & 13666.3191 & 13666.3191 \\
\hline 0 & 0 & 0 & 11670.1175 & 0 & 0 & 0 & 11670.1175 & 11670.1175 \\
\hline 0 & 0 & 0 & 1901.7093 & 0 & 0 & 0 & 1901.7093 & 1901.7093 \\
\hline 0 & 0 & 0 & 0 & 0 & 0 & 0 & 0 & \\
\hline 0 & 0 & 0 & 5768.6186 & 0 & 0 & 0 & 5768.6186 & 5768.6186 \\
\hline 0 & 0 & 0 & 0 & 0 & 0 & 0 & 0 & \\
\hline 0 & 0 & 0 & 0 & 0 & 0 & 0 & 0 & \\
\hline 0 & 0.0001 & 0 & 0 & 0.0282 & 0.008 & 0 & 0.0201 & 0.0282 \\
\hline 0 & 0 & 0 & 0 & 0 & 0 & 0 & 0 & \\
\hline 0 & 0 & 0 & 0 & 0 & 0 & 0 & 0 & \\
\hline 0 & 0 & 0 & 1895.8035 & 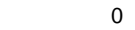 & 0 & 0 & 1895.8035 & 1895.8035 \\
\hline 0 & 0 & 0 & 2267.8775 & 0 & 0 & 0 & 2267.8775 & 2267.8775 \\
\hline 0 & 0 & 0 & 0 & 0 & 0 & 0 & 0 & \\
\hline 0 & 0 & 0 & 3390.0019 & 0 & 0 & 0 & 3390.0019 & 3390.0019 \\
\hline 0 & 1.1023 & 0.0009 & 0 & 26.0112 & 6.7131 & 0.8402 & 18.4579 & 26.0112 \\
\hline 0 & 391.1629 & 70051.4378 & 11438.9706 & 32763.9479 & 8704.2143 & 769.1693 & 34751.6747 & 44202.9202 \\
\hline 0 & 4.1699 & 0 & 0 & 39.0794 & 10.5845 & 0.8129 & 27.682 & 39.0794 \\
\hline 0 & 0 & 0 & 0 & 0 & 0 & 0 & 0 & \\
\hline 0 & 168469 & 0.0039 & 0 & 0 & 0 & 0 & 0 & \\
\hline 0 & 17.9352 & 0.0033 & 0 & 0 & 0 & 0 & 0 & \\
\hline 0 & 44890.871 & 0.0382 & 0 & 0 & 0 & 0 & 0 & \\
\hline 0 & 0 & 0 & 0 & 0 & 0 & 0 & 0 & \\
\hline 0 & 0.0009 & 0.005 & 0 & 1.3482 & 0 & 0 & 1.3482 & 1.3482 \\
\hline 0 & 2.0409 & 0 & 0 & 722.5768 & 191.184 & 15.5354 & 515.8574 & 722.5768 \\
\hline 0 & 0 & 0 & 0 & 0 & 0 & 0 & 0 & \\
\hline 0 & 0 & 0 & 0 & 0 & 0 & 0 & 0 & \\
\hline 0 & 0 & 0 & 0 & 0 & 0 & 0 & 0 & \\
\hline 0 & 0 & 0 & 0 & 0 & 0 & 0 & 0 & \\
\hline 0 & 0 & 0 & 0 & 0 & 0 & 0 & 0 & \\
\hline 0 & 0 & 0 & 0 & 0 & 0 & 0 & 0 & \\
\hline 0 & 0 & 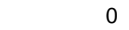 & 0 & 0 & 0 & 0 & 0 & \\
\hline 0 & 0 & 0 & 0 & 0 & 0 & 0 & 0 & \\
\hline
\end{tabular}

\begin{tabular}{|c|c|c|c|c|c|}
\hline 107 & 108 & 109 & 125 & 211 & 212 \\
\hline & \multicolumn{2}{|c|}{ Water Losses Underflow } & \multicolumn{2}{|r|}{ From A500 } & SulfuricAcid \\
\hline 20 & -27.7742 & -27.7742 & 96.2098 & 74 & 20 \\
\hline .0132 & 1.01 & 1.0 & 48 & .0397 & 3.4474 \\
\hline 66 & 4.04 & -3.3724 & 916 & -334.29 & -3.653 \\
\hline 0 & 0 & 0 & 1 & 0 & 0 \\
\hline 22.14 & 9.758 & 764.2178 & 142.716 & 77487.0003 & 1641.1199 \\
\hline
\end{tabular}




\begin{tabular}{|c|c|c|c|c|c|c|c|c|c|c|c|c|c|c|c|}
\hline Stream No. & 214 & 215 & 216 & 217 & 218 & 219 & 220 & 222 & 227 & 228 & 229 & 230 & 232 & 233 & 238 \\
\hline Stream Name & & P STM & HP STM & & & ecycle Watr & & & $\mathrm{a}(\mathrm{OH}) 2$ & & o Landfill & & & uricAcid & \\
\hline Temp C & 73.6046 & 163.2652 & 267 & 190 & 101.1 & 36.7532 & 101.1 & 50 & 20 & 50.0325 & 52.5328 & 52.5652 & 50.5251 & 20 & 49.991 \\
\hline Pres bar & 3.0397 & 4.4786 & 13.1722 & 38.7669 & 1.0132 & 1.0132 & 1.0132 & 1 & 1.0132 & 2.0265 & 1.0132 & 2.0265 & 2.0265 & 3.4474 & 2.0265 \\
\hline Enth MW & -337.94 & -32.867 & -105.1 & -738.38 & -144.88 & -688.79 & -586.94 & -1149.4 & -8.6251 & -926.06 & -20.826 & -907.12 & -1270.7 & -1.8954 & -927.96 \\
\hline Vapor mass fraction & 0 & 1 & 1 & 0 & 1 & 0 & 0 & 0 & 0 & 0 & $1.78 \mathrm{E}-06$ & 0 & 0 & 0 & 0 \\
\hline $\begin{array}{l}\text { Total kgh } \\
\text { Fowrates in kg/h }\end{array}$ & 79128.1188 & 8975.0001 & 29042.5201 & 217282.709 & 39835.1765 & 161535 & 177447.532 & 308015.669 & 2331.7899 & 238116 & 6133.3985 & 232834 & 335687.526 & 851.52 & 238967.372 \\
\hline Glucose & 0 & 0 & 0 & 1887.0023 & 0 & 14.8202 & 1887.0023 & 1899.013 & 0 & 1771.9691 & 9.3511 & 1762.618 & 1892.4713 & 0 & 1771.9691 \\
\hline Cellulose & 0 & 0 & 0 & 22223.5898 & 0 & 5.4601 & 22223.5898 & 22228.0159 & 0 & 111.1394 & 110.5837 & 0.5557 & 22118.468 & 0 & 111.1394 \\
\hline Xylose & 0 & 0 & 0 & 13976.8518 & 0 & 163.8025 & 13976.8518 & 14109.602 & 0 & 13169.903 & 69.5009 & 13100.4023 & 14071.1538 & 0 & 13169.903 \\
\hline Xylan & 0 & 0 & 0 & 341.6581 & 0 & 0.78 & 341.6581 & 342.2903 & 0 & 1.7114 & 1.7029 & 0.0086 & 340.7352 & 0 & 1.7114 \\
\hline Lignin & 0 & 0 & 0 & 11086.6117 & 0 & 55.3808 & 11086.6117 & 11131.4934 & 0 & 55.6571 & 55.3788 & 0.2783 & 11086.6135 & 0 & 55.6571 \\
\hline Acetate & 0 & 0 & 0 & 0 & 0 & 0 & 0 & 0 & 0 & 0 & 0 & 0 & 0 & 0 & 0 \\
\hline Organism & 0 & 0 & 0 & 0 & 0 & 106.0816 & 0 & 85.9718 & 0 & 0 & 0 & 0 & 106.0816 & 0 & 0 \\
\hline Solslds & 106.8592 & 0 & 0 & 5875.4781 & 0 & 1723.0453 & 5875.4781 & 7271.887 & 0 & 6861.7527 & 36.2112 & 6825.5415 & 7562.3115 & 0 & 6861.7527 \\
\hline Gypsum & 0 & 0 & 0 & 0 & 0 & 0 & 0 & 0 & 0 & 2728.0646 & 4201.7444 & 21.1142 & 21.1142 & 0 & 2728.0646 \\
\hline Lgnsol & 0 & 0 & 0 & 583.5059 & 0 & 173.9427 & 583.5059 & 724.4744 & 0 & 683.8315 & 3.6087 & 680.2227 & 753.8398 & 0 & 683.8315 \\
\hline HMF & 0 & 0 & 0 & 88.1958 & 0.1608 & 36.6606 & 88.035 & 117.7457 & 0 & 112.9064 & 0.5958 & 112.3106 & 124.0996 & 0 & 112.9064 \\
\hline C5Sugar & 0 & 0 & 0 & 2380.9848 & 0 & 155.2223 & 2380.9848 & 2506.7818 & 0 & 2341.334 & 12.3558 & 2328.9784 & 2523.8513 & 0 & 2341.334 \\
\hline C6Sugar & 0 & 0 & 0 & 2708.2656 & 0 & 443.8268 & 2708.2656 & 3067.9568 & 0 & 2865.4715 & 15.1218 & 2850.3496 & 3136.9709 & 0 & 2865.4715 \\
\hline C5Solid & 0 & 0 & 0 & 222.7605 & 0 & 0 & 222.7605 & 222.7605 & 0 & 0 & 0 & 0 & 222.7605 & 0 & 0 \\
\hline C6Solid & 0 & 0 & 0 & 56.697 & 0 & 0 & 56.697 & 56.697 & 0 & 0 & 0 & 0 & 56.697 & 0 & 0 \\
\hline $\mathrm{CaH} 2 \mathrm{O} 2$ & 0 & 0 & 0 & 0 & 0 & 0 & 0 & 0 & 2331.7899 & 1157.7868 & 511.9401 & 2.5725 & 2.5725 & 0 & 1157.7868 \\
\hline Ash & 0 & 0 & 0 & 3390.0019 & 0 & 17.9403 & 3390.0019 & 3404.5414 & 0 & 17.0227 & 16.9376 & 0.0851 & 3391.0043 & 0 & 17.0227 \\
\hline Ethanol & 55.3796 & 0 & 0 & 73.8375 & 58.0866 & 21.8403 & 15.7509 & 32.7729 & 0 & 31.911 & 0.1684 & 31.7426 & 36.7448 & 0 & 31.911 \\
\hline Water & 76639.144 & 8975.0001 & 29042.5201 & 147175.543 & 38990.1268 & 156542.14 & 108185.416 & 234728.608 & 0 & 202007.447 & 1066.0438 & 200941.406 & 263338.068 & 0 & 202007.447 \\
\hline Furfural & 99.8393 & 0 & 0 & 565.9027 & 344.9017 & 234.7835 & 221.001 & 407.3621 & 0 & 392.6971 & 2.0724 & 390.6247 & 449.7974 & 0 & 392.6971 \\
\hline Sulfuric Acid & 1641.1199 & 0 & 0 & 1641.1199 & 0 & 28.0804 & 1641.1199 & 1663.8773 & 0 & 0 & 0 & 0 & 115.1391 & 851.52 & 851.52 \\
\hline Nitrogen & 0 & 0 & 0 & 0 & 0 & 0 & 0 & 1.8626 & 0 & 1.8626 & 0.0098 & 1.8527 & 1.8527 & 0 & 1.8626 \\
\hline Carbon Dioxide & 0 & 0 & 0 & 0 & 0 & 22.6203 & 0 & 0.3966 & 0 & 0.3966 & 0.0021 & 0.3945 & 4.6827 & 0 & 0.3966 \\
\hline Oxygen & 0 & 0 & 0 & 0 & 0 & 0 & 0 & 1.0972 & 0 & 1.0972 & 0.0058 & 1.0914 & 1.0914 & 0 & 1.0972 \\
\hline Ammonia & 0 & 0 & 0 & 0 & 0 & 0 & 0 & 0 & 0 & 0 & 0 & 0 & 0 & 0 & 0 \\
\hline Lactic Acid & 0 & 0 & 0 & 1.3482 & 0.0023 & 742.5714 & 1.3459 & 603.1483 & 0 & 571.7846 & 3.0174 & 568.7671 & 740.8992 & 0 & 571.7846 \\
\hline Acetic Acid & 585.7761 & 0 & 0 & 3003.3522 & 441.8991 & 1045.9956 & 2561.4532 & 3407.3079 & 0 & 3230.1278 & 17.0462 & 3213.0816 & 3588.55 & 0 & 3230.1278 \\
\hline
\end{tabular}




\begin{tabular}{|c|c|c|c|c|c|c|c|c|c|c|c|c|c|c|c|}
\hline Stream No. & 239 & 240 & 250 & 252 & 253 & 255 & 259 & 260 & 261 & 262 & 302 & 303 & 304 & 305 & 308 \\
\hline Stream Name & & & & & & WET SOLIDS & & VET AIR & To combustor & 600 & STM & & & & \\
\hline Temp C & 52.4998 & 50 & 50.4926 & 36.7532 & 36.7532 & 50 & 40 & 50 & 38 & 38 & 163.26 & 147.741 & 41 & 65 & 41 \\
\hline Pres bar & 1.0132 & 1 & 1 & 1.0132 & 1.0132 & 1 & 9.6259 & 1 & 1 & 1 & 4.4786 & 4.4786 & 1.0132 & 2.0265 & 1.0132 \\
\hline Enth MW & -927.96 & -916.35 & -1270.7 & -558.21 & -130.57 & -233 & -0.0016604 & -1.2258 & -0.65558 & -0.68983 & -29.141 & -33.91 & -4.0549 & -1265.9 & -43.769 \\
\hline Vapor mass fraction & $1.77 \mathrm{E}-06$ & $1.76 \mathrm{E}-06$ & 0 & 0 & 0 & 0 & 1 & 1 & 1 & 0 & 1 & 0.0003125 & 1 & 0 & 1 \\
\hline $\begin{array}{l}\text { Total kg/h } \\
\text { Fowrates in } \mathrm{kg} / \mathrm{h}\end{array}$ & 238967.372 & 235784.061 & 335687.526 & 130913 & 30622.0067 & 72231.587 & 3744.6703 & 4089.5237 & 3931.4011 & 158.1227 & 7957.6491 & 7957.6491 & 1637.2007 & 335687.526 & 17656.106 \\
\hline Glucose & 1771.9691 & 1771.9691 & 1892.4713 & 12.0108 & 2.8095 & 127.044 & 0 & 0 & 0 & 0 & 0 & 0 & 0 & 1892.4713 & 0 \\
\hline Cellulose & 111.1394 & 111.1394 & 22118.468 & 4.425 & 1.0351 & 22116.8769 & 0 & 0 & 0 & 0 & 0 & 0 & 0 & 22118.468 & 0 \\
\hline xylose & 13169.903 & 13169.903 & 14071.1538 & 132.7506 & 31.0519 & 939.6995 & 0 & 0 & 0 & 0 & 0 & 0 & 0 & 14071.1538 & 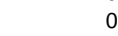 \\
\hline Xylan & 1.7114 & 1.7114 & 340.7352 & 0.6321 & 0.1479 & 340.5788 & 0 & 0 & 0 & 0 & 0 & 0 & 0 & 340.7352 & 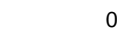 \\
\hline Lignin & 55.6571 & 55.6571 & 11086.6135 & 44.8823 & 10.4985 & 11075.8362 & 0 & 0 & 0 & 0 & 0 & 0 & 0 & 11086.6135 & 0 \\
\hline Acetate & 0 & 0 & 0 & 0 & 0 & 0 & 0 & 0 & 0 & 0 & 0 & 0 & 0 & 0 & 0 \\
\hline Organism & 0 & 0 & 106.0816 & 85.9718 & 20.1098 & 85.9718 & 0 & 0 & 0 & 0 & 0 & 0 & 0 & 106.0816 & 0 \\
\hline Solslds & 6861.7527 & 6861.7527 & 7562.3115 & 1396.4096 & 326.6358 & 410.1344 & 0 & 0 & 0 & 0 & 0 & 0 & 0 & 7562.3115 & 0 \\
\hline Gypsum & 4222.8586 & 0 & 21.1142 & 0 & 0 & 0 & 0 & 0 & 0 & 0 & 0 & 0 & 0 & 21.1142 & 0 \\
\hline Lgnsol & 683.8315 & 683.8315 & 753.8398 & 140.9685 & 32.9741 & 40.643 & 0 & 0 & 0 & 0 & 0 & 0 & 0 & 753.8398 & 0 \\
\hline HMF & 112.9064 & 112.9064 & 124.0996 & 29.7109 & 6.9497 & 4.8393 & 0 & 0.0001 & 0 & 0.0001 & 0 & 0 & 0 & 124.0996 & 0.0001 \\
\hline C5Sug & 2341.334 & 2341.334 & 2523.8513 & 125.797 & 29.4253 & 165.4476 & 0 & 0 & . & 0 & 0 & 0 & 0 & 2523.8513 & 0 \\
\hline C6Sugar & 2865.4715 & 2865.4719 & 3136.9709 & 359.691 & 84.1357 & 202.4852 & 0 & 0 & 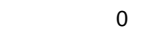 & 0 & 0 & 0 & 0 & 3136.9709 & 0 \\
\hline C5Solid & 0 & 0 & 222.7605 & 0 & 0 & 222.7605 & 0 & 0 & 0 & 0 & 0 & 0 & 0 & 222.7605 & . \\
\hline C6Solid & 0 & 0 & 56.697 & 0 & 0 & 56.697 & 0 & 0 & 0 & 0 & 0 & 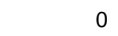 & 0 & 56.697 & 0 \\
\hline $\mathrm{CaH} 2 \mathrm{O} 2$ & 514.5127 & 0 & 2.5725 & 0 & 0 & 0 & 0 & 0 & 0 & 0 & 0 & 0 & 0 & 2.5725 & 0 \\
\hline Ash & 17.0227 & 17.0227 & 3391.0043 & 14.5394 & 3.4009 & 3387.5186 & 0 & 0 & 0 & 0 & 0 & 0 & 0 & 3391.0043 & 0 \\
\hline Ethanol & 31.911 & 31.911 & 36.7448 & 17.7001 & 4.1402 & 0.8619 & 0 & 0.6781 & 0.643 & 0.0351 & 0 & 0 & 41.8156 & 36.7448 & 525.2215 \\
\hline Water & 202007.447 & 202007.447 & 263338.068 & 126866.625 & 29675.5171 & 32721.1684 & 0 & 323.4317 & 166.9911 & 156.4406 & 7957.6491 & 7957.6491 & 52.606 & 263338.068 & 565.3127 \\
\hline Furfural & 392.697 & 392.6971 & 449.7974 & 190.2759 & 44.5076 & 14.665 & 0 & 3.9147 & 3.6061 & 0.3087 & 0 & 0 & 0.5099 & 449.7974 & 5.3992 \\
\hline Sulfuric Acid & 0 & 1554.0614 & 115.1391 & 22.7572 & 5.3232 & 109.8159 & 0 & 0 & 0 & 0 & 0 & 0 & 0 & 115.1391 & 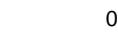 \\
\hline Nitroge & 1.8626 & 1.8626 & 1.8527 & 0 & 0 & 0 & 2872.4811 & 2870.6182 & 2870.6167 & 0.0016 & 0 & 0 & 0.1852 & 1.8527 & 1.667 \\
\hline Carbon Dioxide & 0.3966 & 0.3966 & 4.6827 & 18.3322 & 4.2881 & 0 & 0 & 17.9356 & 17.9352 & 0.0004 & 0 & 0 & 1534.2887 & 4.6827 & 16522.6496 \\
\hline Oxygen & 1.0972 & 1.0972 & 1.0914 & 0 & 0 & 0 & 872.1891 & 871.0919 & 871.091 & 0.0009 & 0 & 0 & 7.523 & 1.0914 & 32.9163 \\
\hline Ammonia & 0 & 0 & 0 & 0 & 0 & 0 & 0 & 0 & 0 & 0 & 0 & 0 & 0 & 0 & 0 \\
\hline Lactic Acid & 571.7845 & 571.7846 & 740.8992 & 1.803 & 140.7684 & 31.3637 & 0 & 0.0005 & 0 & 0.0005 & 0 & 0 & 0.0001 & 740.8992 & 0.0017 \\
\hline Acetic Acid & 3230.1278 & 3230.1281 & 3588.55 & 847.7074 & 198.2882 & 177.18 & 0 & 1.8528 & 0.5181 & 1.3347 & 0 & 0 & 0.2721 & 3588.55 & 2.94 \\
\hline
\end{tabular}




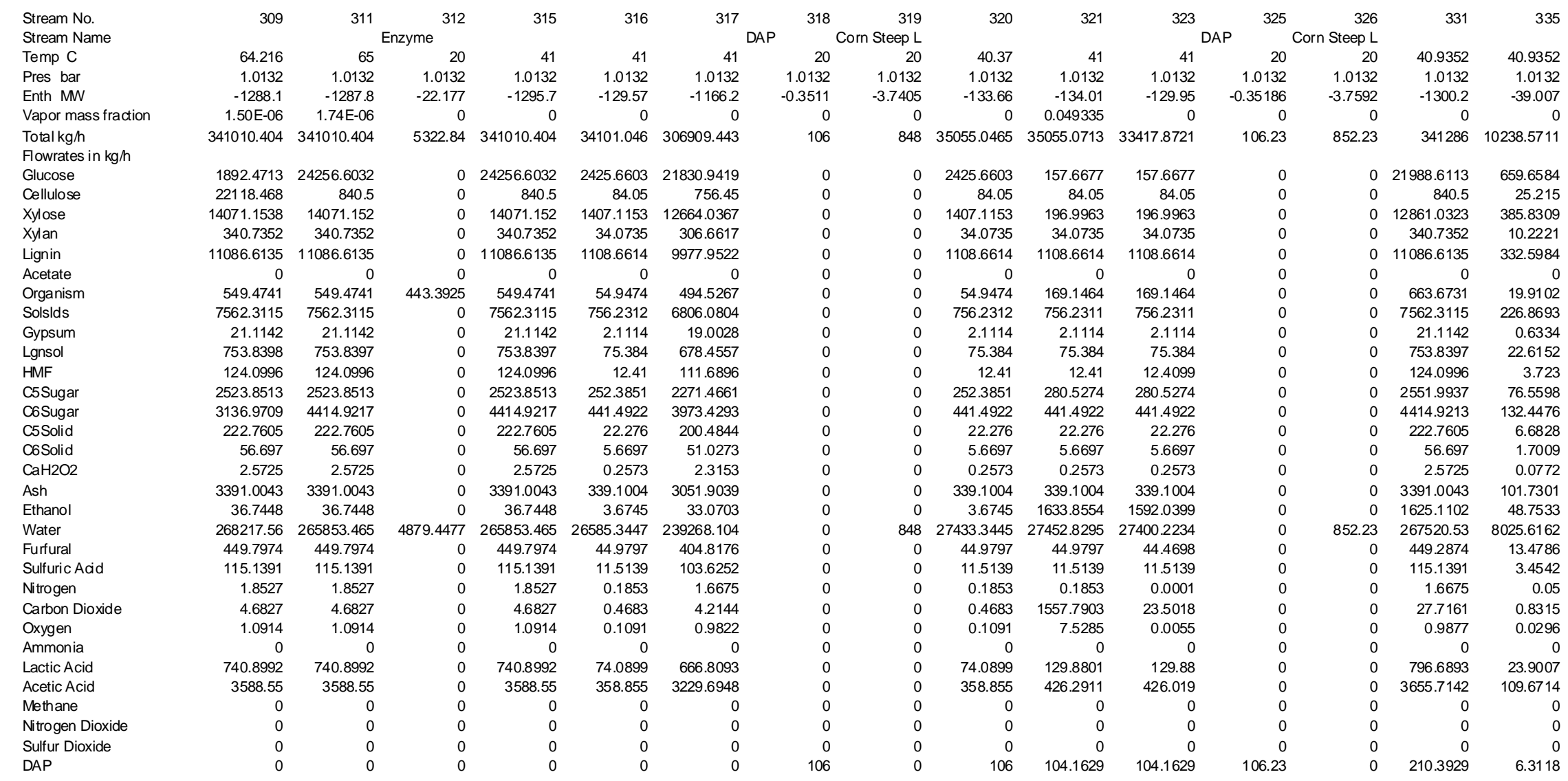




\begin{tabular}{|c|c|c|c|c|c|c|c|c|c|c|c|c|c|c|c|}
\hline $\begin{array}{l}\text { Stream No. } \\
\text { Stream Name }\end{array}$ & 336 & 337 & 338 & 501 & 502 & 506 & 508 & 510 & 511 & 512 & 515 & 516 & 517 & 518 & 519 \\
\hline Temp C & 40.9352 & 41 & 51.697 & 95 & 41 & 100 & 99.0442 & 113.2729 & 91.7119 & 116 & 37.1918 & 120.4596 & 35 & 116.7672 & 91.7 \\
\hline Pres bar & 1.0132 & 1.0132 & 1.0132 & 4.053 & 1.0132 & 4.8231 & 1.8892 & 1.9384 & 1.6779 & 1.6779 & 4.053 & 4.3468 & 1.6779 & 2.1305 & 1.4591 \\
\hline Enth MW & -1261.2 & $\begin{array}{r}-39.192 \\
-392\end{array}$ & -1261.2 & -1368.4 & -1260.6 & -1366.5 & -3.5616 & -134.34 & -40.942 & -40.644 & -32.075 & -125.44 & -16.751 & -1199.7 & -26.878 \\
\hline Vapor mass fraction & 0 & 0 & 0.059362 & 0.00040053 & 0 & 0.00039771 & 1 & 1 & 0.99992 & 1 & 0 & 0 & 0 & 0 & 1 \\
\hline $\begin{array}{l}\text { Total kg/h } \\
\text { Fowrates in } \mathrm{kg} / \mathrm{h}\end{array}$ & 331047.191 & 10238.5711 & 331047.304 & 353760 & 323630 & 353760 & 1660.1368 & 48616.7744 & 25831.903 & 25831.903 & 19091.7542 & 29525.0963 & 6740.1483 & 303483 & 19091.7542 \\
\hline Glucose & 21328.9533 & 0 & 63.9846 & 63.9846 & 63.9846 & 63.9846 & 0 & 0 & 0 & 0 & 0 & 0 & 0 & 63.9846 & 0 \\
\hline Cellulose & 815.285 & 25.215 & 815.285 & 840.5 & 840.5 & 840.5 & 0 & 0 & 0 & 0 & 0 & 0 & 0 & 840.5 & 0 \\
\hline xylose & 12475.2023 & 0 & 748.5122 & 748.5122 & 748.5122 & 748.5122 & 0 & 0 & 0 & 0 & 0 & 0 & 0 & 748.5122 & 0 \\
\hline Xylan & 330.5132 & 10.2221 & 330.5132 & 340.7353 & 340.7353 & 340.7353 & 0 & 0 & 0 & 0 & 0 & 0 & 0 & 340.7353 & 0 \\
\hline Lignin & 10754.0151 & 332.5984 & 10754.0151 & 11086.6135 & 11086.6135 & 11086.6135 & 0 & 0 & 0 & 0 & 0 & 0 & 0 & 11086.6135 & 0 \\
\hline Acetate & 0 & 0 & 0 & 0 & 0 & 0 & 0 & 0 & 0 & 0 & 0 & 0 & 0 & 0 & 0 \\
\hline Organism & 643.763 & 19.9102 & 1135.5483 & 1155.4586 & 1155.4586 & 1155.4586 & 0 & 0 & 0 & 0 & 0 & 0 & 0 & 1155.4586 & 0 \\
\hline Solslds & 7335.4422 & 226.8693 & 7335.4422 & 7562.3115 & 7562.3115 & 7562.3115 & 0 & 0 & 0 & 0 & 0 & 0 & 0 & 7562.3115 & 0 \\
\hline Gypsum & 20.4808 & 0.6334 & 20.4808 & 21.1142 & 21.1142 & 21.1142 & 0 & 0 & 0 & 0 & 0 & 0 & 0 & 21.1142 & 0 \\
\hline Lgnsol & 731.2246 & 22.6152 & 731.2246 & 753.8397 & 753.8397 & 753.8397 & 0 & 0 & 0 & 0 & 0 & 0 & 0 & 753.8397 & 0 \\
\hline HMF & 120.3766 & 3.723 & 120.3766 & 124.0996 & 124.0995 & 124.0996 & 0 & 0.0888 & 0 & 0 & 0 & 0.0888 & 0 & 124.0108 & 0 \\
\hline C5Sugar & 2475.4338 & 50.5295 & 1068.9459 & 1119.4754 & 1119.4754 & 1119.4754 & 0 & 0 & 0 & 0 & 0 & 0 & 0 & 1119.4754 & 0 \\
\hline C6Sugar & 4282.4736 & 75.4951 & 2890.6695 & 2966.165 & 2966.165 & 2966.165 & 0 & 0 & 0 & 0 & 0 & 0 & 0 & 2966.165 & 0 \\
\hline C5Solid & 216.0777 & 6.6828 & 216.0777 & 222.7605 & 222.7605 & 222.7605 & 0 & 0 & 0 & 0 & 0 & 0 & 0 & 222.7605 & 0 \\
\hline C6Solid & 54.9961 & 1.7009 & 54.9961 & 56.697 & 56.697 & 56.697 & 0 & 0 & 0 & 0 & 0 & 0 & 0 & 56.697 & 0 \\
\hline $\mathrm{CaH} 2 \mathrm{O} 2$ & 2.4954 & 0.0772 & 2.4954 & 2.5726 & 2.5726 & 2.5726 & 0 & 0 & 0 & 0 & 0 & 0 & 0 & 2.5726 & 0 \\
\hline Ash & 3289.2742 & 101.7301 & 3289.2742 & 3391.0043 & 3391.0043 & 3391.0043 & 0 & 0 & 0 & 0 & 0 & 0 & 0 & 3391.0043 & 0 \\
\hline Ethanol & 1576.357 & 48.7533 & 19086.9508 & 20125.6153 & 18610.4821 & 20125.6153 & 976.4801 & 18997.3946 & 23698.6457 & 23698.6457 & 18982.6157 & 14.7626 & 4716.0304 & 151.743 & 18982.6157 \\
\hline Water & 259495 & 8025.6162 & 259579 & 295624.461 & 267039.155 & 295624.461 & 431.215 & 29201.9543 & 2132.0895 & 2132.0895 & 107.9716 & 29094.0773 & 2024.1179 & 265991.385 & 107.9716 \\
\hline Furfural & 435.8088 & 13.4786 & 435.8088 & 455.2627 & 443.8883 & 455.2627 & 5.4887 & 236.1783 & 0.0591 & 0.0591 & 0.0591 & 236.1192 & 0 & 213.5969 & 0.0591 \\
\hline Sulfuric Acid & 111.6849 & 3.4542 & 111.6849 & 115.139 & 115.139 & 115.139 & 0 & 0 & 0 & 0 & 0 & 0 & 0 & 115.139 & 0 \\
\hline Nitrogen & 1.6175 & 0.05 & 1.6175 & 0.0006 & 0.0006 & 0.0006 & 0.0006 & 0 & 0 & 0 & 0 & 0 & 0 & 0 & 0 \\
\hline Carbon Dioxide & 26.8847 & 0.8315 & 16754.8623 & 247.2211 & 233.0453 & 247.2211 & 246.1115 & 1.1096 & 1.1096 & 1.1096 & 1.1096 & 0 & 0 & 0 & 1.1096 \\
\hline Oxygen & 0.9581 & 0.0296 & 32.9088 & 0.024 & 0.0222 & 0.024 & 0.0239 & 0 & 0 & 0 & 0 & 0 & 0 & 0 & 0 \\
\hline Ammonia & 0 & 0 & 0 & 0 & 0 & 0 & 0 & 0 & 0 & 0 & 0 & 0 & 0 & 0 & 0 \\
\hline Lactic Acid & 772.7886 & 1152.3726 & 1117.6733 & 2270.0462 & 2270.0444 & 2270.0462 & 0.0001 & 1.3676 & 0 & 0 & 0 & 1.3676 & 0 & 2268.6792 & 0 \\
\hline Acetic Acid & 3546.043 & 109.6714 & 4152.9097 & 4263.6704 & 4259.6412 & 4263.6704 & 0.8169 & 178.6808 & 0 & 0 & 0 & 178.6808 & 0 & 4084.1732 & 0 \\
\hline Methane & 0 & 0 & 0 & 0 & 0 & 0 & 0 & 0 & 0 & 0 & 0 & 0 & 0 & 0 & 0 \\
\hline Nitrogen Dioxide & 0 & 0 & 0 & 0 & 0 & 0 & 0 & 0 & 0 & 0 & 0 & 0 & 0 & 0 & 0 \\
\hline Sulfur Dioxide & 0 & 0 & 0 & 0 & 0 & 0 & 0 & 0 & 0 & 0 & 0 & 0 & 0 & 0 & 0 \\
\hline DAP & 204.0811 & 6.3118 & 196.1697 & 202.4815 & 202.4815 & 202.4815 & 0 & 0 & 0 & 0 & 0 & 0 & 0 & 202.4815 & 0 \\
\hline
\end{tabular}




\begin{tabular}{|c|c|c|c|c|c|c|c|c|c|c|c|c|c|c|c|}
\hline $\begin{array}{l}\text { Stream Name } \\
\text { Temp C }\end{array}$ & 116 & 49.2528 & 26 & 87.0895 & 87.0895 & 86.487 & 71.0548 & 71.0548 & 71.2455 & 63.3463 & 63.3463 & 62.5145 & 74.1511 & 74.1511 & 40 \\
\hline Pres bar & 1.6779 & 1.0132 & 1.0132 & 0.6181 & 0.6181 & 0.6181 & 0.3192 & 0.3192 & 0.3293 & 0.2232 & 0.2232 & 0.2232 & 2.0265 & 2.0265 & 3.2424 \\
\hline Enth MW & -26.666 & -51.385 & -123.36 & -916.78 & -249.61 & -292.67 & -525.99 & -208.99 & -245.49 & -275.26 & -214.23 & -251.91 & -790.07 & -69.605 & -119.57 \\
\hline Vapor mass fraction & 1 & 0.99936 & 0 & $2.90 \mathrm{E}-06$ & 1 & $7.02 \mathrm{E}-06$ & 0 & 1 & 1.15E-05 & 0 & 1 & 4.69E-05 & 0 & 0 & 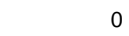 \\
\hline $\begin{array}{l}\text { Total kg/h } \\
\text { Fowrates in } \mathrm{kg} / \mathrm{h}\end{array}$ & 19091.7542 & 20953.3948 & 28011.3594 & 235470.714 & 68012.2636 & 68012.2636 & 137879.196 & 56740.7874 & 56740.7874 & 79735.2309 & 58144 & 58144 & 182897.019 & 16113.0238 & 33192.2135 \\
\hline Glucose & 0 & 0 & 0 & 63.9846 & 0 & 0 & 56.8544 & 0 & 0 & 56.8544 & 0 & 0 & 0 & 0 & 4.8928 \\
\hline Cellulose & 0 & 0 & 0 & 840.5 & 0 & 0 & 16.1736 & 0 & 0 & 16.1736 & 0 & 0 & 0 & 0 & 823.69 \\
\hline xylose & 0 & 0 & 0 & 748.5121 & 0 & 0 & 648.1553 & 0 & 0 & 648.1553 & 0 & 0 & 0 & 0 & 74.8512 \\
\hline Xylan & 0 & 0 & 0 & 340.7353 & 0 & 0 & 6.5567 & 0 & 0 & 6.5567 & 0 & 0 & 0 & 0 & 333.9206 \\
\hline Lignin & 0 & 0 & 0 & 11086.6135 & 0 & 0 & 213.3372 & 0 & 0 & 213.3372 & 0 & 0 & 0 & 0 & 10864.8812 \\
\hline Acetate & 0 & 0 & 0 & 0 & 0 & 0 & 0 & 0 & 0 & 0 & 0 & 0 & 0 & 0 & 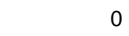 \\
\hline Organism & 0 & 0 & 0 & 1155.4586 & 0 & 0 & 555.8557 & 0 & 0 & 555.8557 & 0 & 0 & 0 & 0 & 577.7293 \\
\hline Solslds & 0 & 0 & 0 & 7562.3115 & 0 & 0 & 6719.6051 & 0 & 0 & 6719.6051 & 0 & 0 & 0 & 0 & 578.2827 \\
\hline Gypsum & 0 & 0 & 0 & 21.1142 & 0 & 0 & 0.4063 & 0 & 0 & 0.4063 & 0 & 0 & 0 & 0 & 20.6919 \\
\hline Lgnsol & 0 & 0 & 0 & 753.8397 & 0 & 0 & 669.8356 & 0 & 0 & 669.8356 & 0 & 0 & 0 & 0 & 57.6454 \\
\hline HMF & 0 & 0.0001 & 0 & 123.844 & 0.1668 & 0.1668 & 109.942 & 0.1014 & 0.1014 & 109.8329 & 0.1091 & 0.1091 & 0.3774 & 0.0332 & 9.4702 \\
\hline C5Sugar & 0 & 0 & 0 & 1119.4754 & 0 & 0 & 994.7266 & 0 & 0 & 994.7265 & 0 & 0 & 0 & 0 & 85.6052 \\
\hline C6Sugar & 0 & 0 & 0 & 2966.165 & 0 & 0 & 2568.4759 & 0 & 0 & 2568.4759 & 0 & 0 & 0 & 0 & 296.6165 \\
\hline C5Solid & 0 & 0 & 0 & 222.7605 & 0 & 0 & 4.2865 & 0 & 0 & 4.2865 & 0 & 0 & 0 & 0 & 218.3053 \\
\hline C6Solid & 0 & 0 & 0 & 56.697 & 0 & 0 & 1.091 & 0 & 0 & 1.091 & 0 & 0 & 0 & 0 & 55.563 \\
\hline $\mathrm{CaH} 2 \mathrm{O} 2$ & 0 & 0 & 0 & 2.5726 & 0 & 0 & 0 & 0 & 0 & 0 & 0 & 0 & 0 & 0 & 2.5726 \\
\hline Ash & 0 & 0 & 0 & 3391.0043 & 0 & 0 & 65.2523 & 0 & 0 & 65.2523 & 0 & 0 & 0 & 0 & 3323.1843 \\
\hline Ethanol & 18982.6157 & 1543.4823 & 0 & 31.1905 & 120.5525 & 120.5525 & 4.0738 & 23.641 & 23.641 & 0.3324 & 3.7414 & 3.7414 & 147.9348 & 13.0329 & 2.3851 \\
\hline Water & 107.9716 & 1049.1178 & 28011.3594 & 198777.387 & 67214.019 & 67214.019 & 120473.609 & 56153.0132 & 56153.0132 & 63064.5348 & 57409.0707 & 57409.0707 & 180776.106 & 15926.1721 & 15200.3162 \\
\hline Furfural & 0.0591 & 11.3977 & 0 & 70.2409 & 143.356 & 143.356 & 16.5697 & 45.8439 & 45.8439 & 3.1367 & 13.433 & 13.433 & 202.6329 & 17.8517 & 5.3713 \\
\hline Sulfuric Acid & 0 & 0 & 0 & 115.139 & 0 & 0 & 102.3085 & 0 & 0 & 102.3085 & 0 & 0 & 0 & 0 & 8.8046 \\
\hline Nitrogen & 0 & 1.8528 & 0 & 0 & 0 & 0 & 0 & 0 & 0 & 0 & 0 & 0 & 0 & 0 & 0 \\
\hline Carbon Dioxide & 1.1096 & 18303.0492 & 0 & 0 & 0 & 0 & 0 & 0 & 0 & 0 & 0 & 0 & 0 & 0 & 0 \\
\hline Oxygen & 0 & 40.4633 & 0 & 0 & 0 & 0 & 0 & 0 & 0 & 0 & 0 & 0 & 0 & 0 & 0 \\
\hline Ammonia & 0 & 0 & 0 & 0 & 0 & 0 & 0 & 0 & 0 & 0 & 0 & 0 & 0 & 0 & 0 \\
\hline Lactic Acid & 0 & 0.0018 & 0 & 2266.4819 & 2.1972 & 2.1972 & 2012.4651 & 1.4511 & 1.4511 & 2010.4273 & 2.0378 & 2.0378 & 5.6861 & 0.5009 & 173.3158 \\
\hline Acetic Aci & 0 & 4.0289 & 0 & 3552.2003 & 531.9725 & 531.9725 & 2639.6188 & 516.7417 & 516.7417 & 1924.0465 & 715.5725 & 715.5725 & 1764.2867 & 155.4317 & 271.6333 \\
\hline Methane & 0 & 0 & 0 & 0 & 0 & 0 & 0 & 0 & 0 & 0 & 0 & 0 & 0 & 0 & \\
\hline Nitrogen Dioxide & 0 & 0 & 0 & 0 & 0 & 0 & 0 & 0 & 0 & 0 & 0 & 0 & 0 & 0 & - \\
\hline Sulfur Dioxide & 0 & 0 & 0 & 0 & 0 & 0 & 0 & 0 & 0 & 0 & 0 & 0 & 0 & 0 & \\
\hline DAP & 0 & 0 & 0 & 202.4815 & 0 & 0 & 0 & 0 & 0 & 0 & 0 & 0 & 0 & 0 & 202.4815 \\
\hline
\end{tabular}




\begin{tabular}{|c|c|c|c|c|c|c|c|c|c|c|c|c|c|c|c|}
\hline Stream No. & 550 & 551 & 552 & 557 & 558 & 559 & 560 & 571 & 572 & 573 & 574 & 575 & 576 & 579 & 580 \\
\hline Stream Name & Vent & & & & & & From A500 & WET SOLIDS & & & A900 Water & EVAP BTMS & & o 800 & \\
\hline Temp C & 34.171 & 50.4061 & 41.8714 & 25 & 404.576 & 40 & 40 & 40 & 40 & 40 & 20 & 63.4809 & 41.9386 & 40 & 40 \\
\hline Pres bar & 0.9119 & 0.9119 & 4.053 & 1.0132 & 9.6317 & 9.6317 & 1.0132 & 1.0132 & 3.2424 & 3.2424 & 1.0132 & 3.2424 & 1.0132 & 1.0132 & 3.2424 \\
\hline Enth MW & -47.109 & -127.64 & -1388.2 & 1.19E-08 & 0.48461 & -0.0020127 & -0.8183 & -118.59 & -808.66 & -30.617 & -462.22 & -275.25 & -618.28 & -0.8183 & -778.05 \\
\hline Vapor mass fraction & 1 & 0 & 0 & 1 & 1 & 1 & 1 & 0 & 0 & 0 & 0 & 0 & 0 & 1 & 0 \\
\hline $\begin{array}{l}\text { Total } \mathrm{kg} / \mathrm{h} \\
\text { Fowrates in } \mathrm{kg} / \mathrm{h}\end{array}$ & 18834.7073 & 30130.0504 & 353760 & 4618.3987 & 4618.3987 & 4618.3987 & 4844.8852 & 32965.7291 & 202278.497 & 7658.512 & 104788.002 & 79735.2309 & 141971.605 & 4844.8852 & 194620 \\
\hline Glucose & 0 & 0 & 63.9846 & 0 & 0 & 0 & 0 & 4.8928 & 59.0917 & 2.2373 & 0 & 56.8544 & 2.2373 & 0 & 56.8544 \\
\hline Cellulose & 0 & 0 & 840.5 & 0 & 0 & 0 & 0 & 823.69 & 16.81 & 0.6364 & 0 & 16.1736 & 0.6364 & 0 & 16.1736 \\
\hline xylose & 0 & 0 & 748.5122 & 0 & 0 & 0 & 0 & 74.8512 & 673.6609 & 25.5056 & 0 & 648.1553 & 25.5056 & 0 & 648.1553 \\
\hline Xylan & 0 & 0 & 340.7353 & 0 & 0 & 0 & 0 & 333.9206 & 6.8147 & 0.258 & 0 & 6.5567 & 0.258 & 0 & 6.5567 \\
\hline Lignin & 0 & 0 & 11086.6135 & 0 & 0 & 0 & 0 & 10864.8812 & 221.7323 & 8.3951 & 0 & 213.3372 & 8.3951 & 0 & 213.3372 \\
\hline Acetate & 0 & 0 & 0 & 0 & 0 & 0 & 0 & 0 & 0 & 0 & 0 & 0 & 0 & 0 & 0 \\
\hline Organism & 0 & 0 & 1155.4586 & 0 & 0 & 0 & 0 & 577.7293 & 577.7293 & 21.8735 & 0 & 555.8557 & 21.8735 & 0 & 555.8557 \\
\hline Solsids & 0 & 0 & 7562.3115 & 0 & 0 & 0 & 0 & 578.2827 & 6984.0291 & 264.4239 & 0 & 6719.6051 & 264.4239 & 0 & 6719.6051 \\
\hline Gypsum & 0 & 0 & 21.1142 & 0 & 0 & 0 & 0 & 20.6919 & 0.4223 & 0.016 & 0 & 0.4063 & 0.016 & 0 & 0.4063 \\
\hline Lgnsol & 0 & 0 & 753.8397 & 0 & 0 & 0 & 0 & 57.6454 & 696.1944 & 26.3588 & 0 & 669.8356 & 26.3588 & 0 & 669.8356 \\
\hline HMF & 0 & 0.0001 & 124.0996 & 0 & 0 & 0 & 0.0001 & 9.4702 & 114.3738 & 4.3303 & 0 & 109.8329 & 4.4191 & 0.0001 & 110.0434 \\
\hline C5Sugar & 0 & 0 & 1119.4754 & 0 & 0 & 0 & 0 & 85.6052 & 1033.8702 & 39.1436 & 0 & 994.7265 & 39.1436 & 0 & 994.7266 \\
\hline C6Sugar & 0 & 0 & 2966.165 & 0 & 0 & 0 & 0 & 296.6165 & 2669.5483 & 101.0724 & 0 & 2568.4759 & 101.0724 & 0 & 2568.4759 \\
\hline C5Solid & 0 & 0 & 222.7605 & 0 & 0 & 0 & 0 & 218.3053 & 4.4552 & 0.1687 & 0 & 4.2865 & 0.1687 & 0 & 4.2865 \\
\hline C6Solid & 0 & 0 & 56.697 & 0 & 0 & 0 & 0 & 55.563 & 1.1339 & 0.0429 & 0 & 1.091 & 0.0429 & 0 & 1.091 \\
\hline $\mathrm{CaH} 2 \mathrm{O} 2$ & 0 & 0 & 2.5726 & 0 & 0 & 0 & 0 & 2.5726 & 0 & 0 & 0 & 0 & 0 & 0 & 0 \\
\hline Ash & 0 & 0 & 3391.0043 & 0 & 0 & 0 & 0 & 3323.1843 & 67.8201 & 2.5678 & 0 & 65.2523 & 2.5678 & 0 & 65.2523 \\
\hline Ethanol & 28.3489 & 1515.1336 & 20125.6153 & 0 & 0 & 0 & 0.4593 & 1.9258 & 28.8054 & 1.0906 & 0 & 0.3324 & 15.8532 & 0.4593 & 27.7148 \\
\hline Water & 475.1486 & 28585.3309 & 295624.461 & 0 & 0 & 0 & 224.1718 & 14976.1441 & 183577.068 & 6950.453 & 104788.002 & 63064.5348 & 140832.536 & 224.1718 & 176626.615 \\
\hline Furfural & 0.0233 & 11.3744 & 455.2627 & 0 & 0 & 0 & 0.5638 & 4.8074 & 64.8697 & 2.456 & 0 & 3.1367 & 238.5753 & 0.5638 & 62.4136 \\
\hline Sulfuric Acid & 0 & 0 & 115.139 & 0 & 0 & 0 & 0 & 8.8046 & 106.3345 & 4.026 & 0 & 102.3085 & 4.026 & 0 & 102.3085 \\
\hline Nitrogen & 1.8527 & 0 & 0.0006 & 3648.5353 & 3648.5353 & 3648.5353 & 3648.3834 & 0.1521 & 0 & 0 & 0 & 0 & 0 & 3648.3834 & 0 \\
\hline Carbon Dioxide & 18288.8727 & 14.1758 & 247.2211 & 0 & 0 & 0 & 0 & 0 & 0 & 0 & 0 & 0 & 0 & 0 & 0 \\
\hline Oxygen & 40.4615 & 0.0018 & 0.024 & 969.8633 & 969.8633 & 969.8633 & 969.7831 & 0.0802 & 0 & 0 & 0 & 0 & 0 & 969.7831 & 0 \\
\hline Ammonia & 0 & 0 & 0 & 0 & 0 & 0 & 0 & 0 & 0 & 0 & 0 & 0 & 0 & 0 & 0 \\
\hline Lactic Acid & 0 & 0.0018 & 2270.0462 & 0 & 0 & 0 & 0.0009 & 173.3149 & 2093.1662 & 79.2498 & 0 & 2010.4273 & 80.6174 & 0.0009 & 2013.9162 \\
\hline Acetic Acid & 0 & 4.0289 & 4263.6704 & 0 & 0 & 0 & 1.5228 & 270.1105 & 3280.5669 & 124.2063 & 0 & 1924.0465 & 302.8871 & 1.5228 & 3156.3608 \\
\hline Methane & 0 & 0 & 0 & 0 & 0 & 0 & 0 & 0 & 0 & 0 & 0 & 0 & 0 & 0 & 0 \\
\hline Nitrogen Dioxide & 0 & 0 & 0 & 0 & 0 & 0 & 0 & 0 & 0 & 0 & 0 & 0 & 0 & 0 & 0 \\
\hline Sulfur Dioxide & 0 & 0 & 0 & 0 & 0 & 0 & 0 & 0 & 0 & 0 & 0 & 0 & 0 & 0 & 0 \\
\hline DAP & 0 & 0 & 202.4815 & 0 & 0 & 0 & 0 & 202.4815 & 0 & 0 & 0 & 0 & 0 & 0 & 0 \\
\hline
\end{tabular}




\begin{tabular}{|c|c|c|c|c|c|c|c|c|c|c|c|c|c|c|}
\hline Stream No. & 596 & 597 & 612 & 614 & 615 & 617 & 618 & 619 & 620 & 621 & 623 & 624 & 625 & 626 \\
\hline Stream Name & LP STM & & To Anaerobic & & From A600 & & & & & To 600 & & & & \\
\hline Temp C & 163.26 & 147.741 & 81.1983 & 81.536 & 81.6342 & 81.536 & 81.5361 & 52.8112 & 21 & 35.0121 & 0 & 21 & 21 & 20 \\
\hline Pres bar & 4.4786 & 4.4786 & 2.0265 & 2.0265 & 2.0265 & 2.0265 & 2.0265 & 1.0132 & 0.9818 & 1 & 0 & 0.9818 & 0.9818 & 1.0132 \\
\hline Enth MW & -1.8203 & -2.1182 & -309.07 & -3.5383 & -3.5433 & -305.53 & -305.53 & -305.57 & -1.3884 & -173.22 & 0 & -308.92 & 0 & -0.043101 \\
\hline Vapor mass fraction & 1 & 0.0003125 & 0 & 1 & 1 & 0 & 0 & 0.33471 & 1 & 0 & 0 & 0 & 0 & 1 \\
\hline $\begin{array}{l}\text { Total } \mathrm{kg} / \mathrm{h} \\
\text { Fowrates in } \mathrm{kg} / \mathrm{h}\end{array}$ & 497.0711 & 497.0711 & 71889.3232 & 1476.6225 & 1478.0052 & 70412.7028 & 70412.7028 & 101106.802 & 31055.3256 & 39993.296 & 0 & 70051.4874 & 0 & 30694.0996 \\
\hline Glucose & 0 & 0 & 0 & 0 & 0 & 0 & 0 & 0 & 0 & 0 & 0 & 0 & 0 & 0 \\
\hline Cellulose & 0 & 0 & 0 & 0 & 0 & 0 & 0 & 0 & 0 & 0 & 0 & 0 & 0 & 0 \\
\hline Xylose & 0 & 0 & 0 & 0 & 0 & 0 & 0 & 0 & 0 & 0 & 0 & 0 & 0 & 0 \\
\hline Xylan & 0 & 0 & 0 & 0 & 0 & 0 & 0 & 0 & 0 & 0 & 0 & 0 & 0 & 0 \\
\hline Lignin & 0 & 0 & 0 & 0 & 0 & 0 & 0 & 0 & 0 & 0 & 0 & 0 & 0 & 0 \\
\hline Acetate & 0 & 0 & 0 & 0 & 0 & 0 & 0 & 0 & 0 & 0 & 0 & 0 & 0 & 0 \\
\hline Organism & 0 & 0 & 0 & 0 & 0 & 0 & 0 & 0 & 0 & 0 & 0 & 0 & 0 & 0 \\
\hline Solslds & 0 & 0 & 0 & 0 & 0 & 0 & 0 & 0 & 0 & 0 & 0 & 0 & 0 & 0 \\
\hline Gypsum & 0 & 0 & 0 & 0 & 0 & 0 & 0 & 0 & 0 & 0 & 0 & 0 & 0 & 0 \\
\hline Lgnsol & 0 & 0 & 0 & 0 & 0 & 0 & 0 & 0 & 0 & 0 & 0 & 0 & 0 & 0 \\
\hline HMF & 0 & 0 & 0.1942 & 0 & 0 & 0 & 0 & 0 & 0 & 0.1609 & 0 & 0 & 0 & 0 \\
\hline C5Sugar & 0 & 0 & 0 & 0 & 0 & 0 & 0 & 0 & 0 & 0 & 0 & 0 & 0 & 0 \\
\hline C6Sugar & 0 & 0 & 0 & 0 & 0 & 0 & 0 & 0 & 0 & 0 & 0 & 0 & 0 & 0 \\
\hline C5Solid & 0 & 0 & 0 & 0 & 0 & 0 & 0 & 0 & 0 & 0 & 0 & 0 & 0 & 0 \\
\hline C6Solid & 0 & 0 & 0 & 0 & 0 & 0 & 0 & 0 & 0 & 0 & 0 & 0 & 0 & 0 \\
\hline $\mathrm{CaH} 2 \mathrm{O} 2$ & 0 & 0 & 0 & 0 & 0 & 0 & 0 & 0 & 0 & 0 & 0 & 0 & 0 & 0 \\
\hline Ash & 0 & 0 & 0 & 0 & 0 & 0 & 0 & 0 & 0 & 0 & 0 & 0 & 0 & 0 \\
\hline Ethanol & 0 & 0 & 71.1546 & 0.062 & 0.0623 & 1.3611 & 1.3611 & 1.3611 & 0.0127 & 58.1217 & 0 & 0.0009 & 0 & 0 \\
\hline Water & 497.0711 & 497.0711 & 70855.7421 & 252.1075 & 253.4381 & 70399.336 & 70399.336 & 70399.336 & 351.4123 & 39146.5666 & 0 & 70051.4378 & 0 & 0 \\
\hline Furfural & 0 & 0 & 363.0621 & 0 & 0 & 0 & 0 & 0 & 0 & 345.2103 & 0 & 0 & 0 & 0 \\
\hline Sulfuric Acid & 0 & 0 & 0 & 0 & 0 & 0 & 0 & 0 & 0 & 0 & 0 & 0 & 0 & 0 \\
\hline Nitrogen & 0 & 0 & 0.0016 & 0.0016 & 0.0016 & 0 & 0 & 23545 & 23545 & 0.0016 & 0 & 0.0039 & 0 & 23545 \\
\hline Carbon Dioxide & 0 & 0 & 0.0004 & 877.1952 & 877.2455 & 10.7803 & 10.7803 & 10.7803 & 16.0637 & 0.0004 & 0 & 0.0033 & 0 & 0 \\
\hline Oxygen & 0 & 0 & 0.0009 & 0.0009 & 0.0009 & 0 & 0 & 7149.1088 & 7142.8502 & 0.0009 & 0 & 0.0382 & 0 & 7149.1092 \\
\hline Ammonia & 0 & 0 & 0 & 0 & 0 & 0 & 0 & 0 & 0 & 0 & 0 & 0 & 0 & 0 \\
\hline Lactic Acid & 0 & 0 & 0.5038 & 0 & 0 & 0.5038 & 0.5038 & 0.5038 & 0 & 0.0029 & 0 & 0.005 & 0 & 0 \\
\hline Acetic Acid & 0 & 0 & 598.6655 & 0 & 0 & 0 & 0 & 0 & 0 & 443.2338 & 0 & 0 & 0 & 0 \\
\hline Methane & 0 & 0 & 0 & 347.2553 & 347.2569 & 0.7222 & 0.7222 & 0.7222 & 0 & 0 & 0 & 0 & 0 & 0 \\
\hline
\end{tabular}


Stream No.

Temp C

Pres bar

Vapor mass fraction

Vapor mass

Total kg/h

Glucose

Cellulose

xylose

Xylan
Lignin

Acetate

Organis

Solslds

Gypsum

Lgns

HMF

C5Sugar

C6Sugar

C5Solid

C6Solid
CaH2O2

Ash

Ethanol

Water

Furfural

Sulfuric Acid

Nitrogen

Carbon Dioxide

Oxygen

Lactic Acid

Acetic Acid

Nitrogen Dioxide

Sulfur Dioxide

DAP

2-2-4-TriMth-C5
$703 \quad 800$

$804 \quad 811 \quad 812$

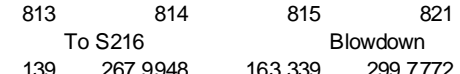

${ }^{823}$ CORN STOVITo S215 ${ }^{842}$ To S302 843

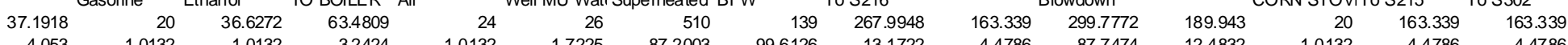

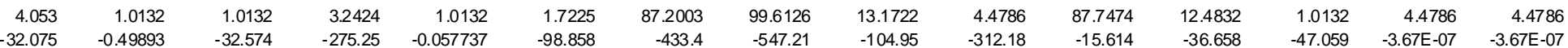

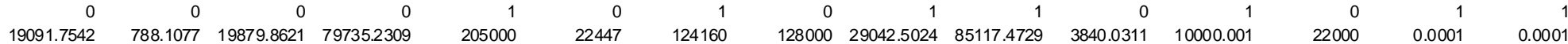

0
0
0
0
0
0
0
0
0
0
0
0
0
0
0
0
0
0
22447
0
0
0
0
0
0
0
0
0
0
0
0
0

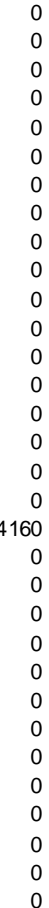

$\begin{array}{rrrr}0 & 0 & 0 & 0 \\ 0 & 6999.0446 & 0 & 0 \\ 0 & 0 & 0 & 0 \\ 0 & 3942.4986 & 0 & 0 \\ 0 & 3366.629 & 0 & 0 \\ 0 & 548.6106 & 0 & 0 \\ 0 & 0 & 0 & 0 \\ 0 & 1664.147 & 0 & 0 \\ 0 & 0 & 0 & 0 \\ 0 & 0 & 0 & 0 \\ 0 & 0 & 0 & 0 \\ 0 & 0 & 0 & 0 \\ 0 & 0 & 0 & 0 \\ 0 & 0 & 0 \\ 0 & 546.9069 & 0 & 0 \\ 0 & 654.2438 & 0 & 0 \\ 0 & 0 & 0 & 0 \\ 0 & 977.9574 & 0 & 0 \\ 0 & 0 & 0 & 0.0001 \\ 0 & 3299.9472 & 0.0001 & 0 \\ 0 & 0 & 0 & 0 \\ 0 & 0 & 0 & 0 \\ 0 & 0 & 0 & 0 \\ 0 & 0 & 0 & 0 \\ 0 & 0 & 0 & 0 \\ 0 & 0 & 0 & 0 \\ 0 & 0 & 0 & 0 \\ 0 & 0 & 0 & 0 \\ 0 & 0 & 0 & 0 \\ 0 & 0 & 0 & 0 \\ 0 & 0 & 0 & 0 \\ 0 & 0 & 0 & 0 \\ 0 & 0 & 0 & 0 \\ 0 & & & \end{array}$




\begin{tabular}{|c|c|c|c|c|c|c|c|c|c|c|c|c|c|c|c|}
\hline Stream No. & 844 & 845 & 846 & 848 & 849 & 850 & 851 & 852 & 854 & 855 & 856 & 861 & 862 & \multirow{2}{*}{\multicolumn{2}{|c|}{$\begin{array}{lr}863 & 864 \\
& \text { Cond 501\&50 }\end{array}$}} \\
\hline Stream Name & To Mol Sve & To D501 & To D502 & \multicolumn{3}{|c|}{ to Dearatr } & \multicolumn{3}{|c|}{ To Evaprator } & \multicolumn{4}{|c|}{ Evap Cond } & & \\
\hline Temp C & 163.339 & 163.339 & 163.339 & 163.339 & 163.339 & 117.9874 & 117.9874 & 117.9874 & 50.7428 & 50.7428 & 50.7581 & 115.21 & 30.2857 & 117.6356 & 147.74 \\
\hline Pres bar & 4.4786 & 4.4786 & 4.4786 & 4.4786 & 4.4786 & 1.7225 & 1.7225 & 1.7225 & 0.1013 & 0.1013 & 4.2556 & 1.7023 & 3.445 & 1.7023 & 4.4786 \\
\hline Enth $\mathrm{MW}$ & -1.8228 & -198.65 & -24.25 & -87.452 & $-3.67 E-08$ & -93.788 & -78.884 & -14.903 & -79.884 & -79.884 & -87.847 & -16.312 & -98.856 & -418.86 & -259.3 \\
\hline Vapor mass fraction & 1 & 1 & 1 & 1 & 1 & 0.60031 & 0.60031 & 0.60031 & 0.60031 & 0.60031 & 0 & 0 & 0 & 0.60031 & 0 \\
\hline Total kg/h & 497 & 54164.0071 & 6612 & 23844.4667 & 0 & 23844.4667 & 20055.4662 & 3789.0001 & 20055.4679 & 20055.4679 & 20055.4679 & 3789.0001 & 22447 & 106484.012 & 60776 \\
\hline \multicolumn{16}{|l|}{ Fowrates in kg/h } \\
\hline Water & 497 & 54164.0071 & 6612 & 23844.4667 & 0 & 23844.4667 & 20055.4662 & 3789.0001 & 20055.4679 & 20055.4679 & 20055.4679 & 3789.0001 & 22447 & 106484.012 & 60776 \\
\hline Stream No. & 865 & 869 & 871 & 872 & 873 & 921 & 922 & 923 & 924 & 925 & 926 & 931 & 932 & 944 & 950 \\
\hline Stream Name & Cond E302 & To WWT & VENT & \multicolumn{2}{|c|}{ BFW } & CWS & \multicolumn{2}{|c|}{ cws } & \multicolumn{2}{|c|}{ cWs } & \multicolumn{2}{|c|}{ cWs } & \multicolumn{3}{|c|}{ CT BLOWDOWN } \\
\hline Temp C & 147.74 & 120.6474 & 137 & 137 & 138.5518 & 28 & 37 & 28 & 37 & 28 & 37 & 28 & 37 & 28 & 44.4833 \\
\hline Pres bar & 4.4786 & 2.0265 & 3.3437 & 3.3437 & 99.6126 & 4.1341 & 4.1341 & 4.1341 & 4.1341 & 4.1341 & 4.1341 & 4.1341 & 4.1341 & 1.0132 & 0.9818 \\
\hline Enth $\mathrm{MW}$ & -38.291 & -10.227 & 0 & -671.03 & -547.28 & -2215 & -2207.3 & 0 & 0 & -50.393 & -50.273 & 0 & 0 & -49.572 & -549.71 \\
\hline Vapor mass fraction & 0 & 0 & 1 & 0 & 0 & 0 & 0 & 0 & 0 & 0 & 0 & 0 & 0 & 0 & 0 \\
\hline Total kg/h & 8975.0001 & 2379.2136 & 0 & 156810 & 128000 & 502646.004 & 502646.004 & 0 & 0 & 11448.3755 & 11448.3755 & 0 & 0 & 11262.0004 & 125794.489 \\
\hline \multicolumn{16}{|l|}{ Flowrates in kg/h } \\
\hline Water & 8975.0001 & 2379.2136 & 0 & 156810 & 128000 & 502646.004 & 502646.004 & 0 & 0 & 11448.3755 & 11448.3755 & 0 & 0 & 11262.0004 & 125148.02 \\
\hline Furfural & 0 & 0 & 0 & 0 & 0 & 0 & 0 & 0 & 0 & 0 & 0 & 0 & 0 & 0 & 61.7581 \\
\hline Sulfuric Acid & 0 & 0 & 0 & 0 & 0 & 0 & 0 & 0 & 0 & 0 & 0 & 0 & 0 & 0 & 0 \\
\hline Nitrogen & 0 & 0 & 0 & 0 & 0 & 0 & 0 & 0 & 0 & 0 & 0 & 0 & 0 & 0 & 0.0039 \\
\hline Carbon Dioxide & 0 & 0 & 0 & 0 & 0 & 0 & 0 & 0 & 0 & 0 & 0 & 0 & 0 & 0 & 0.0033 \\
\hline Oxygen & 0 & 0 & 0 & 0 & 0 & 0 & 0 & 0 & 0 & 0 & 0 & 0 & 0 & 0 & 0.0382 \\
\hline Ammonia & 0 & 0 & 0 & 0 & 0 & 0 & 0 & 0 & 0 & 0 & 0 & 0 & 0 & 0 & 0 \\
\hline Lactic Acid & 0 & 0 & 0 & 0 & 0 & 0 & 0 & 0 & 0 & 0 & 0 & 0 & 0 & 0 & 1.738 \\
\hline Acetic Acid & 0 & 0 & 0 & 0 & 0 & 0 & 0 & 0 & 0 & 0 & 0 & 0 & 0 & 0 & 537.7159 \\
\hline
\end{tabular}




\begin{tabular}{|c|c|c|c|c|c|c|c|c|c|c|c|c|c|c|c|}
\hline $\begin{array}{l}\text { Stream No. } \\
\text { Stream Name }\end{array}$ & CWS & 956 & 957 & 958 & 1003 & 1103 & 1105 & 1110 & 1111 & 1113 & 1114 & 1115 & 1116 & 1120 & 1121 \\
\hline Temp C & 28 & 37 & 28 & 37 & 40 & 241.1346 & 48.8889 & 563.4046 & 499.2643 & 499.2643 & 499.2643 & 499.2643 & 587 & 15.5555 & 68.5937 \\
\hline Pres bar & 4.1341 & 4.1341 & 4.1341 & 4.1341 & 1.0132 & 1.5648 & 1.5648 & 1.5648 & 1.5648 & 1.5648 & 1.5648 & 1.5648 & 1.5648 & 1.0342 & 1.5648 \\
\hline Enth MW & -2190 & -2184.8 & -15872 & -15834 & -0.8183 & -116.5 & -59.05 & -1184.2 & -1184.2 & -1161.3 & -9.7811 & -1151.5 & -1124.6 & -0.29371 & 1.3027 \\
\hline Vapor mass fraction & 0 & 0 & 0 & 0 & 1 & 1 & 0 & 0.72911 & 0.77193 & 0 & 0 & 0 & 0 & 1 & 1 \\
\hline $\begin{array}{l}\text { Total kg/h } \\
\text { Fowrates in kg/h }\end{array}$ & 497538.44 & 497538.44 & 3605820.07 & 3605820.07 & 4844.8852 & 129827.222 & 19281.2176 & 920480 & 920480 & 909549 & 9548.9486 & 900000 & 900000 & 110000 & 110000 \\
\hline Glucose & 0 & 0 & 0 & 0 & 0 & 0 & 4.8928 & 4.8928 & 0.0001 & 0.0001 & 0.0001 & 0 & 0 & 0 & 0 \\
\hline Cellulose & 0 & 0 & 0 & 0 & 0 & 0 & 823.69 & 823.69 & 0.0022 & 0.0022 & 0.0022 & 0 & 0 & 0 & 0 \\
\hline Xylose & 0 & 0 & 0 & 0 & 0 & 0 & 74.8512 & 74.8513 & 0.0002 & 0.0002 & 0.0002 & 0 & 0 & 0 & 0 \\
\hline xylan & 0 & 0 & 0 & 0 & 0 & 0 & 333.9206 & 333.9206 & 0.0008 & 0.0008 & 0.0008 & 0 & 0 & 0 & 0 \\
\hline Lignin & 0 & 0 & 0 & 0 & 0 & 0 & 10864.8812 & 10864.8812 & 0.0275 & 0.0275 & 0.0275 & 0 & 0 & 0 & 0 \\
\hline Acetate & 0 & 0 & 0 & 0 & 0 & 0 & 0 & 0 & 0 & 0 & 0 & 0 & 0 & 0 & 0 \\
\hline Organism & 0 & 0 & 0 & 0 & 0 & 0 & 577.7293 & 577.7293 & 0.0014 & 0.0014 & 0.0014 & 0 & 0 & 0 & 0 \\
\hline Solslds & 0 & 0 & 0 & 0 & 0 & 0.0004 & 578.2827 & 578.2827 & 0.0016 & 0.0016 & 0.0016 & 0 & 0 & 0 & 0 \\
\hline Gypsum & 0 & 0 & 0 & 0 & 0 & 0 & 20.6919 & 20.6919 & 20.6919 & 20.6919 & 20.6919 & 0 & 0 & 0 & 0 \\
\hline Lgnsol & 0 & 0 & 0 & 0 & 0 & 0 & 57.6454 & 57.6454 & 0.0002 & 0.0002 & 0.0002 & 0 & 0 & 0 & 0 \\
\hline HMF & 0 & 0 & 0 & 0 & 0.0001 & 0 & 9.4702 & 9.4702 & 0 & 0 & 0 & 0 & 0 & 0 & 0 \\
\hline C5Sugar & 0 & 0 & 0 & 0 & 0 & 0 & 85.6052 & 85.6053 & 0.0003 & 0.0003 & 0.0003 & 0 & 0 & 0 & 0 \\
\hline C6Sugar & 0 & 0 & 0 & 0 & 0 & 0 & 296.6165 & 296.6165 & 0.0008 & 0.0008 & 0.0008 & 0 & 0 & 0 & 0 \\
\hline C5Solid & 0 & 0 & 0 & 0 & 0 & 0 & 218.3053 & 218.3053 & 0.0005 & 0.0005 & 0.0005 & 0 & 0 & 0 & 0 \\
\hline C6Solid & 0 & 0 & 0 & 0 & 0 & 0 & 55.563 & 55.563 & 0.0001 & 0.0001 & 0.0001 & 0 & 0 & 0 & 0 \\
\hline $\mathrm{CaH} 2 \mathrm{O} 2$ & 0 & 0 & 0 & 0 & 0 & 0 & 2.5726 & 2.5726 & 2.5726 & 2.5726 & 2.5726 & 0 & 0 & 0 & 0 \\
\hline Ash & 0 & 0 & 0 & 0 & 0 & 0 & 3323.1843 & 3323.1843 & 3323.1843 & 3323.1843 & 3323.1843 & 0 & 0 & 0 & 0 \\
\hline Ethanol & 0 & 0 & 0 & 0 & 0.4593 & 1.7278 & 0.1981 & 0.1981 & 0.1981 & 0 & 0 & 0 & 0 & 0 & 0 \\
\hline Water & 497538.44 & 497538.44 & 3605820.07 & 3605820.07 & 224.1718 & 18199.5168 & 1540.2177 & 1540.2177 & 2434.2451 & 0 & 0 & 0 & 0 & 0 & 0 \\
\hline Furfural & 0 & 0 & 0 & 0 & 0.5638 & 4.313 & 0.4944 & 0.4944 & 0.4944 & 0 & 0 & 0 & 0 & 0 & 0 \\
\hline Sulfuric Acid & 0 & 0 & 0 & 0 & 0 & 0 & 8.8046 & 8.8046 & 8.8046 & 0 & 0 & 0 & 0 & 0 & 0 \\
\hline Nitrogen & 0 & 0 & 0 & 0 & 3648.3834 & 84475.2995 & 0.0156 & 95.9293 & 191.8275 & 0 & 0 & 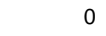 & 0 & 84379.386 & 84379.386 \\
\hline Carbon Dioxide & 0 & 0 & 0 & 0 & 0 & 22960.5251 & 0 & 0 & 1299.9962 & 0 & 0 & 0 & 0 & 0 & 0 \\
\hline Oxygen & 0 & 0 & 0 & 0 & 969.7831 & 3906.8611 & 0.0082 & 0.0165 & 0.0165 & 0 & 0 & 0 & 0 & 25620.614 & 25620.614 \\
\hline Ammonia & 0 & 0 & 0 & 0 & 0 & 0 & 0 & 0 & 0 & 0 & 0 & 0 & 0 & 0 & 0 \\
\hline Lactic Acid & 0 & 0 & 0 & 0 & 0.0009 & 0 & 173.3149 & 173.3149 & 173.3149 & 0 & 0 & 0 & 0 & 0 & 0 \\
\hline Acetic Acid & 0 & 0 & 0 & 0 & 1.5228 & 242.3311 & 27.7795 & 27.7795 & 377.3149 & 0 & 0 & 0 & 0 & 0 & 0 \\
\hline Methane & 0 & 0 & 0 & 0 & 0 & 0.0001 & 0 & 199.9984 & 399.9978 & 0 & 0 & 0 & 0 & 0 & 0 \\
\hline Nitrogen Dioxide & 0 & 0 & 0 & 0 & 0 & 0.4508 & 0 & 0 & 0 & 0 & 0 & 0 & 0 & 0 & 0 \\
\hline Sulfur Dioxide & 0 & 0 & 0 & 0 & 0 & 7.6998 & 0 & 0 & 0 & 0 & 0 & 0 & 0 & 0 & 0 \\
\hline DAP & 0 & 0 & 0 & 0 & 0 & 0 & 202.4815 & 202.4815 & 202.4815 & 202.4815 & 202.4815 & 0 & 0 & 0 & 0 \\
\hline 2-2-4-TriMth-C5 & 0 & 0 & 0 & 0 & 0 & 0 & 0 & 0 & 0 & 0 & 0 & 0 & 0 & 0 & 0 \\
\hline Hydrogen & 0 & 0 & 0 & 0 & 0 & 0.2433 & 0 & 602.1655 & 1204.333 & 0 & 0 & 0 & 0 & 0 & 0 \\
\hline Carbon & 0 & 0 & 0 & 0 & 0 & 0 & 0 & 0 & 5999.983 & 5999.983 & 5999.983 & 0 & 0 & 0 & 0 \\
\hline Carbon Monoxide & 0 & 0 & 0 & 0 & 0 & 28.2569 & 0 & 299.9981 & 599.9972 & 0 & 0 & 0 & 0 & 0 & 0 \\
\hline Ethane & 0 & 0 & 0 & 0 & 0 & 0 & 0 & 0 & 199.9994 & 0 & 0 & 0 & 0 & 0 & 0 \\
\hline Propane & 0 & 0 & 0 & 0 & 0 & 0 & 0 & 0 & 0 & 0 & 0 & 0 & 0 & 0 & 0 \\
\hline HydroxyAcetone & 0 & 0 & 0 & 0 & 0 & 0 & 0 & 0 & 0 & 0 & 0 & 0 & 0 & 0 & 0 \\
\hline Formaldehyde & 0 & 0 & 0 & 0 & 0 & 0 & 0 & 0 & 0 & 0 & 0 & 0 & 0 & 0 & 0 \\
\hline 2-5-Xylenol & 0 & 0 & 0 & 0 & 0 & 0 & 0 & 0 & 0 & 0 & 0 & 0 & 0 & 0 & 0 \\
\hline Sulphur & 0 & 0 & 0 & 0 & 0 & 0 & 0 & 0 & 0.5245 & 0 & 0 & 0 & 0 & 0 & 0 \\
\hline Hydroxyacetal de & 0 & 0 & 0 & 0 & 0 & 0 & 0 & 0 & 1006.6621 & 0 & 0 & 0 & 0 & 0 & 0 \\
\hline Pyrolignin & 0 & 0 & 0 & 0 & 0 & 0 & 0 & 0 & 2516.6585 & 0 & 0 & 0 & 0 & 0 & 0 \\
\hline Levoglucosan & 0 & 0 & 0 & 0 & 0 & 0 & 0 & 0 & 223.7027 & 0 & 0 & 0 & 0 & 0 & 0 \\
\hline Toluene & 0 & 0 & 0 & 0 & 0 & 0 & 0 & 0 & 292.2676 & 0 & 0 & 0 & 0 & 0 & 0 \\
\hline Silicon Dioxide & 0 & 0 & 0 & 0 & 0 & 0 & 0 & 900000 & 900000 & 900000 & 0 & 900000 & 900000 & 0 & 0 \\
\hline
\end{tabular}




\begin{tabular}{|c|c|c|c|c|c|c|c|c|c|c|c|c|c|c|c|}
\hline $\begin{array}{l}\text { Stream No. } \\
\text { Stream Name }\end{array}$ & 1123 & 1124 & 1126 & 1127 & 1130 & 1131 & 1132 & 1133 & 1134 & 1135 & 1137 & 1138 & 1139 & 1140 & 1144 \\
\hline Temp C & 105.3141 & 1596.2098 & 963.8307 & 530 & 499.2643 & 68 & 68.0491 & 68.0491 & 68.0491 & 65.5555 & 65.5555 & 45 & 65.5555 & 68 & 68 \\
\hline Pres bar & 1.5648 & 1.5648 & 1.5648 & 1.5648 & 1.5648 & 1.5648 & 2.7369 & 2.7369 & 2.7369 & 2.7369 & 2.7369 & 2.3922 & 2.7369 & 1.5648 & 1.5648 \\
\hline Enth $\mathrm{MW}$ & -9.0077 & 3.9088 & -39.804 & -56.964 & -22.917 & -566.35 & 4.54E-06 & -566.11 & -566.11 & -566.31 & -549.32 & -550.86 & -16.989 & -10.346 & -10.203 \\
\hline Vapor mass fraction & 1 & 0 & 1 & 1 & 0.77193 & 0 & 0 & 0 & 0 & 0 & 0 & 0 & 0 & 1 & 0.55095 \\
\hline $\begin{array}{l}\text { Total } \mathrm{kg} / \mathrm{h} \\
\text { Fowrates in } \mathrm{kg} / \mathrm{h}\end{array}$ & 120747.026 & 4604.2961 & 116142.716 & 116142.716 & 10930.3544 & 165107.24 & 0.5245 & 165039.655 & 165039.13 & 165039.13 & 160088 & 160088 & 4951.1738 & 5911.0784 & 3514.9112 \\
\hline Glucose & 0.0001 & 0 & 0 & 0 & 0 & 0 & 0 & 0 & 0 & 0 & 0 & 0 & 0 & 0 & 0 \\
\hline Cellulose & 0.0022 & 0.0004 & 0 & 0 & 0 & 0 & 0 & 0 & 0 & 0 & 0 & 0 & 0 & 0 & 0 \\
\hline xylose & 0.0002 & 0 & 0 & 0 & 0 & 0 & 0 & 0 & 0 & 0 & 0 & 0 & 0 & 0 & 促 \\
\hline Xylan & 0.0008 & 0.0003 & 0 & 0 & 0 & 0 & 0 & 0 & 0 & 0 & 0 & 0 & 0 & 0 & 0 \\
\hline Lignin & 0.0275 & 0.0001 & 0 & 0 & 0 & 0 & 0 & 0 & 0 & 0 & 0 & 0 & 0 & 0 & م \\
\hline Acetate & 0 & 0 & 0 & 0 & 0 & 0 & 0 & 0 & 0 & 0 & 0 & 0 & 0 & 0 & 0 \\
\hline Organism & 0.0014 & 0.0001 & 0 & 0 & 0 & 0 & 0 & 0 & 0 & 0 & 0 & 0 & 0 & 0 & 0 \\
\hline Solslds & 0.0016 & 0 & 0.0004 & 0.0004 & 0 & 0 & 0 & 0 & 0 & 0 & 0 & 0 & 0 & 0 & 0 \\
\hline Gypsum & 20.6919 & 0.0007 & 0 & 0 & 0 & 0 & 0 & 0 & 0 & 0 & 0 & 0 & 0 & 0 & 0 \\
\hline Lgnsol & 0.0002 & 0 & 0 & 0 & 0 & 0 & 0 & 0 & 0 & 0 & 0 & 0 & 0 & 0 & 0 \\
\hline HMF & 0 & 0 & 0 & 0 & 0 & 5.4077 & 0 & 5.575 & 5.575 & 5.575 & 5.4077 & 5.4077 & 0.1672 & 0 & 0 \\
\hline C5Sugar & 0.0003 & 0 & 0 & 0 & 0 & 0 & 0 & 0 & 0 & 0 & 0 & 0 & 0 & 0 & 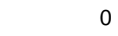 \\
\hline C6Sugar & 0.0008 & 0 & 0 & 0 & 0 & 0 & 0 & 0 & 0 & 0 & 0 & 0 & 0 & 0 & 0 \\
\hline C5Solid & 0.0005 & 0.0005 & 0 & 0 & 0 & 0 & 0 & 0 & 0 & 0 & 0 & 0 & 0 & 0 & 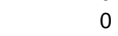 \\
\hline C6Solid & 0.0001 & 0 & 0 & 0 & 0 & 0 & 0 & 0 & 0 & 0 & 0 & 0 & 0 & 0 & 0 \\
\hline $\mathrm{CaH} 2 \mathrm{O} 2$ & 2.5726 & 4400.2646 & 0 & 0 & 0 & 0 & 0 & 0 & 0 & 0 & 0 & 0 & 0 & 0 & 0 \\
\hline Ash & 3323.1843 & 1.5477 & 0 & 0 & 0 & 0 & 0 & 0 & 0 & 0 & 0 & 0 & 0 & 0 & 0 \\
\hline Ethanol & 0 & 0 & 0 & 0 & 0.1981 & 0.7751 & 0 & 0.7748 & 0.7748 & 0.7748 & 0.7516 & 0.7516 & 0.0232 & 0.1745 & 0.1745 \\
\hline Water & 0 & 0 & 4763.5916 & 4763.5916 & 2434.2451 & 31221.3936 & 0 & 31216.7478 & 31216.7478 & 31216.7478 & 30280.2497 & 30280.2497 & 936.5025 & 1493.0971 & 1493.0971 \\
\hline Furfural & 0 & 0 & 0 & 0 & 0.4944 & 10.2535 & 0 & 10.2486 & 10.2486 & 10.2486 & 9.9411 & 9.9411 & 0.3075 & 0.182 & 0.182 \\
\hline Sulfuric Acid & 0 & 0 & 0 & 0 & 8.8046 & 293.4858 & 0 & 293.4859 & 293.4859 & 293.4859 & 284.6813 & 284.6813 & 8.8046 & 0 & 0 \\
\hline Nitrogen & 84475.2995 & 0 & 84475.1648 & 84475.1648 & 191.8275 & 0.0075 & 0 & 0.0075 & 0.0075 & 0.0075 & 0.0072 & 0.0072 & 0.0002 & 191.8273 & 0 \\
\hline Carbon Dioxide & 0 & 0 & 22960.5251 & 22960.5251 & 1299.9962 & 1.089 & 0 & 1.0887 & 1.0887 & 1.0887 & 1.0561 & 1.0561 & 0.0327 & 1299.9632 & 1299.9632 \\
\hline Oxygen & 25620.6228 & 0 & 3906.7875 & 3906.7875 & 0.0165 & 0 & 0 & 0 & 0 & 0 & 0 & 0 & 0 & 0.0165 & 0 \\
\hline Ammonia & 0 & 0 & 0 & 0 & 0 & 0 & 0 & 0 & 0 & 0 & 0 & 0 & 0 & 0 & 0 \\
\hline Lactic Acid & 0 & 0 & 0 & 0 & 173.3149 & 5765.2605 & 0 & 5765.2733 & 5765.2733 & 5765.2733 & 5592.3155 & 5592.3155 & 172.9582 & 0.369 & 0.369 \\
\hline Acetic Acid & 0 & 0 & 0 & 0 & 377.3149 & 8194.0744 & 0 & 8190.1622 & 8190.1622 & 8190.1622 & 7944.4577 & 7944.4577 & 245.7049 & 127.6979 & 127.6979 \\
\hline Methane & 199.9983 & 0 & 0.0001 & 0.0001 & 399.9978 & 0.0376 & 0 & 0.0376 & 0.0376 & 0.0376 & 0.0364 & 0.0364 & 0.0011 & 399.9966 & 0 \\
\hline Nitrogen Dioxide & 0 & 0 & 0.4508 & 0.4508 & 0 & 0 & 0 & 0 & 0 & 0 & 0 & 0 & 0 & 0 & 0 \\
\hline Sulfur Dioxide & 0 & 0 & 7.6998 & 7.6998 & 0 & 0 & 0 & 0 & 0 & 0 & 0 & 0 & 0 & 0 & 0 \\
\hline DAP & 202.4815 & 202.4815 & 0 & 0 & 0 & 0 & 0 & 0 & 0 & 0 & 0 & 0 & 0 & 0 & 0 \\
\hline 2-2-4-Trimth-C5 & 0 & 0 & 0 & 0 & 0 & 0 & 0 & 0 & 0 & 0 & 0 & 0 & 0 & 0 & 0 \\
\hline Hydrogen & 602.1654 & 0 & 0.2433 & 0.2433 & 1204.333 & 0.0731 & 0 & 0.0731 & 0.0731 & 0.0731 & 0.0709 & 0.0709 & 0.0022 & 1204.3308 & 0 \\
\hline Carbon & 5999.983 & 0.0001 & 0 & 0 & 0 & 0 & 0 & 0 & 0 & 0 & 0 & 0 & 0 & 0 & 0 \\
\hline Carbon Monoxide & 299.9981 & 0 & 28.2569 & 28.2569 & 599.9972 & 0.0343 & 0 & 0.0343 & 0.0343 & 0.0343 & 0.0333 & 0.0333 & 0.001 & 599.9962 & 0 \\
\hline Ethane & 0 & 0 & 0 & 0 & 199.9994 & 0.0171 & 0 & 0.0171 & 0.0171 & 0.0171 & 0.0166 & 0.0166 & 0.0005 & 199.9989 & 199.9989 \\
\hline Propane & 0 & 0 & 0 & 0 & 0 & 0 & 0 & 0 & 0 & 0 & 0 & 0 & 0 & 0 & 0 \\
\hline HydroxyAcetone & 足 & 0 & 足 & 0 & 0 & 0 & 0 & 0 & 0 & 0 & 0 & 0 & 0 & 0 & 0 \\
\hline Formaldehyde & 0 & 0 & 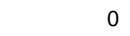 & 0 & 0 & 0 & 0 & 0 & 0 & 0 & 0 & 0 & 0 & 0 & 0 \\
\hline 2-5-Xylenol & 0 & 0 & 0 & 0 & 0 & 0 & 0 & 0 & 0 & 0 & 0 & 0 & 0 & 0 & 0 \\
\hline Sulphur & 0 & 0 & 0 & 0 & 0.5245 & 0.5245 & 0.5245 & 0.5245 & 0 & 0 & 0 & 0 & 0 & 0 & 0 \\
\hline Hydroxyacetalde & 0 & 0 & 0 & 0 & 1006.6621 & 29589.168 & 0 & 29578.3208 & 29578.3208 & 29578.3208 & 28691 & 28691 & 887.3496 & 108.4655 & 108.4655 \\
\hline Pyrolignin & 0 & 0 & 0 & 0 & 2516.6585 & 82451.3562 & 0 & 82406.9041 & 82406.9041 & 82406.9041 & 79934.6981 & 79934.6981 & 2472.2073 & 0 & 0 \\
\hline Levoglucosan & 0 & 0 & 0 & 0 & 223.7027 & 7171.6759 & 0 & 7168.0897 & 7168.0897 & 7168.0897 & 6953.0475 & 6953.0475 & 215.0427 & 5.0733 & 5.0733 \\
\hline Toluene & 0 & 0 & 0 & 0 & 292.2676 & 402.5874 & 0 & 402.2781 & 402.2781 & 402.2781 & 390.2097 & 390.2097 & 12.0683 & 279.89 & 279.89 \\
\hline Silicon Dioxide & 0 & 0 & 0 & 0 & 0 & 0 & 0 & 0 & 0 & 0 & 0 & 0 & 0 & 0 & \\
\hline
\end{tabular}




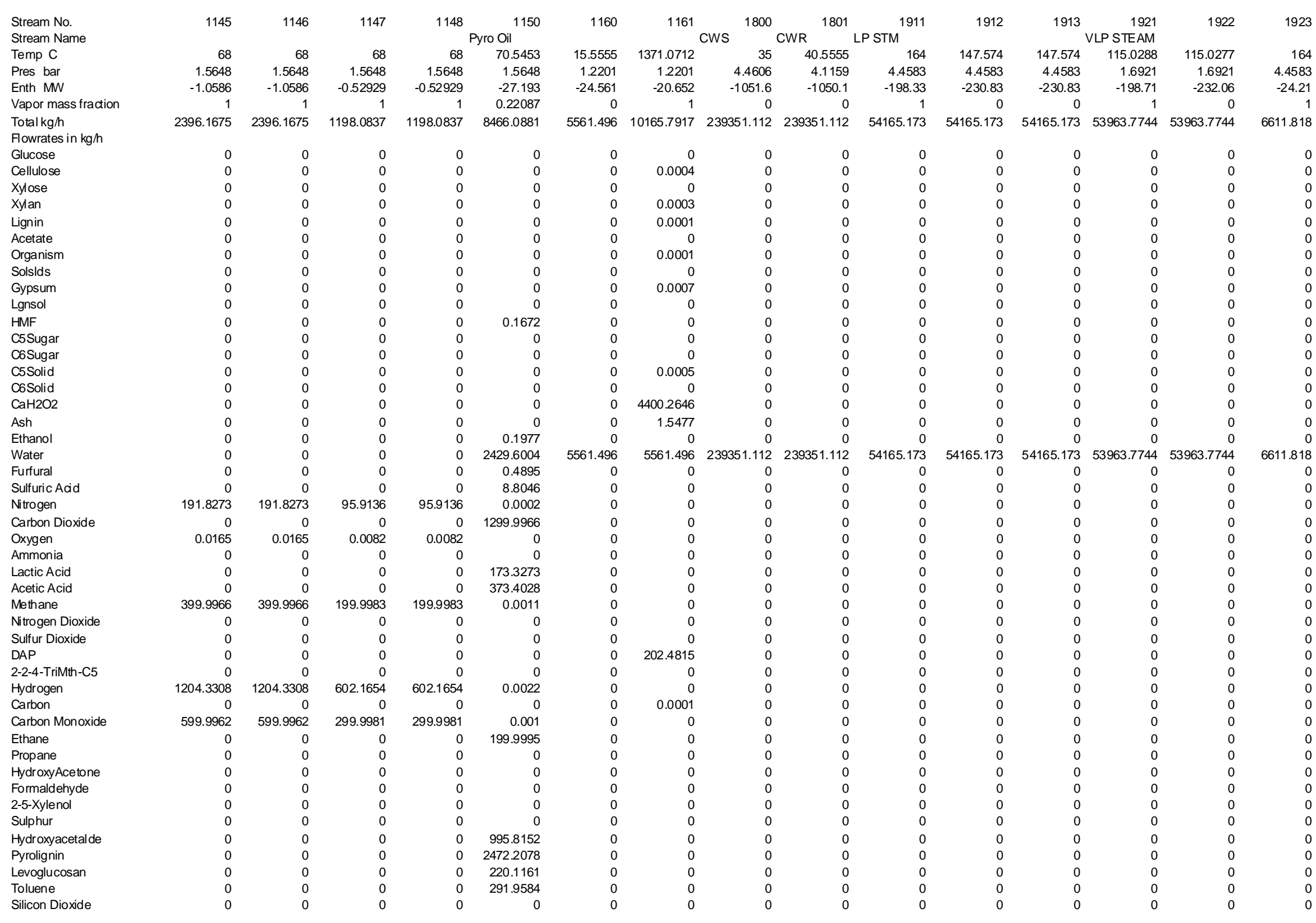




\begin{tabular}{|c|c|c|c|c|c|c|c|c|c|c|c|c|c|c|c|}
\hline Stream No. & 1924 & 1925 & 2217 & 2227 & 2228 & 2230 & 2239 & 2240 & 2241 & 2520 & 2521 & 2522 & 5102 & 5211 & 5219 \\
\hline Stream Name & & & & & & & & FILTRATE & H-244 Vapor & & & & To S102 & To S211 & To S219 \\
\hline Temp C & 147.574 & 147.574 & 197.7722 & 49.8868 & 50 & 52.5328 & 52.5328 & 50 & 35.0121 & 99.8733 & 35 & 35.0121 & 74.1511 & 74.1511 & 41.9386 \\
\hline Pres bar & 4.4583 & 4.4583 & 39.7802 & 1 & 1.0132 & 1.0132 & 2.0265 & 1 & 1 & 1.0132 & 1.0132 & 1 & 2.0265 & 2.0265 & 1.0132 \\
\hline Enth MW & -28.21 & -28.21 & -736.32 & -924.98 & -926.07 & -907.13 & -927.95 & -916.35 & -173.22 & -164.81 & -172.53 & -173.22 & -144.94 & -334.73 & -618.28 \\
\hline Vapor mass fraction & 0 & 0 & 0 & $1.73 E-06$ & 0 & $1.78 \mathrm{E}-06$ & 0 & $1.76 \mathrm{E}-06$ & 0 & 0.19512 & 0 & 0 & 0 & 0 & 0 \\
\hline $\begin{array}{l}\text { Total kg/h } \\
\text { Fowrates in kg/h }\end{array}$ & 6611.818 & 6611.818 & 217282.68 & 238116 & 238116 & 232834 & 238967.372 & 235784.061 & 39993.296 & 39835.1765 & 39835.1765 & 39993.296 & 33553 & 77488 & 141971.605 \\
\hline Glucose & 0 & 0 & 0 & 1771.9691 & 1771.9691 & 1762.618 & 1771.9691 & 1771.9691 & 0 & 0 & 0 & 0 & 0 & 0 & 2.2373 \\
\hline Cellulose & 0 & 0 & 24261.5609 & 111.1394 & 111.1394 & 0.5557 & 111.1394 & 111.1394 & 0 & 0 & 0 & 0 & 0 & 0 & 0.6364 \\
\hline Xylose & 0 & 0 & 0 & 13169.903 & 13169.903 & 13100.4023 & 13169.903 & 13169.903 & 0 & 0 & 0 & 0 & 0 & 0 & 25.5056 \\
\hline Xylan & 0 & 0 & 13666.3191 & 1.7114 & 1.7114 & 0.0086 & 1.7114 & 1.7114 & 0 & 0 & 0 & 0 & 0 & 0 & 0.258 \\
\hline Lignin & 0 & 0 & 11670.1175 & 55.6571 & 55.6571 & 0.2783 & 55.6571 & 55.6571 & 0 & 0 & 0 & 0 & 0 & 0 & 8.3951 \\
\hline Acetate & 0 & 0 & 1901.7093 & 0 & 0 & 0 & 0 & 0 & 0 & 0 & 0 & 0 & 0 & 0 & 0 \\
\hline Organism & 0 & 0 & 0 & 0 & 0 & 0 & 0 & 0 & 0 & 0 & 0 & 0 & 0 & 0 & 21.8735 \\
\hline Solslds & 0 & 0 & 5875.4781 & 6861.7527 & 6861.7527 & 6825.5415 & 6861.7527 & 6861.7527 & 0 & 0 & 0 & 0 & 0 & 0 & 264.4239 \\
\hline Gypsum & 0 & 0 & 0 & 0 & 2728.0646 & 21.1142 & 4222.8586 & 0 & 0 & 0 & 0 & 0 & 0 & 0 & 0.016 \\
\hline Lgnsol & 0 & 0 & 0 & 683.8315 & 683.8315 & 680.2227 & 683.8315 & 683.8315 & 0 & 0 & 0 & 0 & 0 & 0 & 26.3588 \\
\hline HMF & 0 & 0 & 0.0201 & 112.9064 & 112.9064 & 112.3106 & 112.9064 & 112.9064 & 0.1609 & 0.1608 & 0.1608 & 0.1609 & 0.0692 & 0.1599 & 4.4191 \\
\hline C5Sugar & 0 & 0 & 0 & 2341.334 & 2341.334 & 2328.9784 & 2341.334 & 2341.334 & 0 & 0 & 0 & 0 & 0 & 0 & 39.1436 \\
\hline C6Sugar & 0 & 0 & 0 & 2865.4715 & 2865.4715 & 2850.3496 & 2865.4715 & 2865.4719 & 0 & 0 & 0 & 0 & 0 & 0 & 101.0724 \\
\hline C5Solid & 0 & 0 & 1895.8035 & 0 & 0 & 0 & 0 & 0 & 0 & 0 & 0 & 0 & 0 & 0 & 0.1687 \\
\hline C6Solid & 0 & 0 & 2267.8775 & 0 & 0 & 0 & 0 & 0 & 0 & 0 & 0 & 0 & 0 & 0 & 0.0429 \\
\hline $\mathrm{CaH} 2 \mathrm{O} 2$ & 0 & 0 & 0 & 2331.7899 & 1157.7868 & 2.5725 & 514.5127 & 0 & 0 & 0 & 0 & 0 & 0 & 0 & 0 \\
\hline Ash & 0 & 0 & 3390.0019 & 17.0227 & 17.0227 & 0.0851 & 17.0227 & 17.0227 & 0 & 0 & 0 & 0 & 0 & 0 & 2.5678 \\
\hline Ethanol & 0 & 0 & 73.8375 & 31.911 & 31.911 & 31.7426 & 31.911 & 31.911 & 58.1217 & 58.0866 & 58.0866 & 58.1217 & 27.1391 & 62.6756 & 15.8532 \\
\hline Water & 6611.818 & 6611.818 & 149408.337 & 202007.447 & 202007.447 & 200941.406 & 202007.447 & 202007.447 & 39146.5666 & 38990.1268 & 38990.1268 & 39146.5666 & 33163.9136 & 76589.4331 & 140832.536 \\
\hline Furfural & 0 & 0 & 127.5213 & 392.6971 & 392.6971 & 390.6247 & 392.697 & 392.6971 & 345.2103 & 344.9017 & 344.9017 & 345.2103 & 37.1736 & 85.8495 & 238.5753 \\
\hline Sulfuric Acid & 0 & 0 & 1641.1199 & 1554.0614 & 0 & 0 & 0 & 1554.0614 & 0 & 0 & 0 & 0 & 0 & 0 & 4.026 \\
\hline Nitrogen & 0 & 0 & 0 & 1.8626 & 1.8626 & 1.8527 & 1.8626 & 1.8626 & 0.0016 & 0 & 0 & 0.0016 & 0 & 0 & 0 \\
\hline Carbon Dioxide & 0 & 0 & 0 & 0.3966 & 0.3966 & 0.3945 & 0.3966 & 0.3966 & 0.0004 & 0 & 0 & 0.0004 & 0 & 0 & 0 \\
\hline Oxygen & 0 & 0 & 0 & 1.0972 & 1.0972 & 1.0914 & 1.0972 & 1.0972 & 0.0009 & 0 & 0 & 0.0009 & 0 & 0 & 0 \\
\hline Ammonia & 0 & 0 & 0 & 0 & 0 & 0 & 0 & 0 & 0 & 0 & 0 & 0 & 0 & 0 & 0 \\
\hline Lactic Acid & 0 & 0 & 1.3482 & 571.7846 & 571.7846 & 568.7671 & 571.7845 & 571.7846 & 0.0029 & 0.0023 & 0.0023 & 0.0029 & 1.0431 & 2.409 & 80.6174 \\
\hline Acetic Acid & 0 & 0 & 1101.6335 & 3230.1278 & 3230.1278 & 3213.0816 & 3230.1278 & 3230.1281 & 443.2338 & 441.8991 & 441.8991 & 443.2338 & 323.6636 & 747.4755 & 302.8871 \\
\hline
\end{tabular}




\begin{tabular}{|c|c|c|c|c|c|c|c|c|c|c|c|c|c|c|c|}
\hline Stream No. & 5251 & 5511 & 5518 & 5519 & 5520 & 5521 & 5523 & 5525 & 5571 & 5618 & 6110 & 6111 & $\begin{array}{r}6112 \\
\end{array}$ & 8001 & 8002 \\
\hline Temp C & 74.1511 & 122.534 & 86.9859 & 116 & 37 & 70.9641 & 70.9722 & 87.1343 & 63.4809 & 120.3871 & 137 & 179.8844 & 179.8844 & 43.3225 & 59.4266 \\
\hline Pres bar & 2.0265 & 2.1305 & 0.6181 & 1.6779 & 1.0132 & 1.5513 & 1.7225 & 2.0265 & 3.2424 & 2.0272 & 10 & 10 & 10 & 1.0132 & 1.0132 \\
\hline Enth MW & -240.8 & -1197.8 & -1199.7 & -13.978 & -32.077 & -16.538 & -16.538 & -916.77 & -275.25 & -125.45 & -123.14 & -111.95 & -105.98 & -325.85 & -333.71 \\
\hline Vapor mass fraction & 0 & 0 & 0.053693 & 1 & 0 & 0 & 0 & 0 & 0 & $2.59 \mathrm{E}-06$ & 0 & 0.60031 & 0.97 & 0.014868 & 0.041851 \\
\hline $\begin{array}{l}\text { Total } \mathrm{kg} / \mathrm{h} \\
\text { Fowrates in } \mathrm{kg} / \mathrm{h}\end{array}$ & 55743.0011 & 303483 & 303483 & 6740.1483 & 19091.7542 & 6740.1483 & 6740.1483 & 235470.714 & 79735.2309 & 29525.0963 & 28809.6111 & 28809.6111 & 28809.6093 & 103213.215 & 103213.215 \\
\hline Glucose & 0 & 63.9846 & 63.9846 & 0 & 0 & 0 & 0 & 63.9846 & 56.8544 & 0 & 0 & 0 & 0 & 56.8544 & 56.8544 \\
\hline Cellulose & 0 & 840.5 & 840.5 & 0 & 0 & 0 & 0 & 840.5 & 16.1736 & 0 & 0 & 0 & 0 & 7015.2184 & 7015.2184 \\
\hline Xylose & 0 & 748.5122 & 748.5121 & 0 & 0 & 0 & 0 & 748.5121 & 648.1553 & 0 & 0 & 0 & 0 & 648.1553 & 648.1553 \\
\hline Xylan & 0 & 340.7353 & 340.7353 & 0 & 0 & 0 & 0 & 340.7353 & 6.5567 & 0 & 0 & 0 & 0 & 3949.0553 & 3949.0553 \\
\hline Lignin & 0 & 11086.6135 & 11086.6135 & 0 & 0 & 0 & 0 & 11086.6135 & 213.3372 & 0 & 0 & 0 & 0 & 3579.966 & 3579.966 \\
\hline Acetate & 0 & 0 & 0 & 0 & 0 & 0 & 0 & 0 & 0 & 0 & 0 & 0 & 0 & 548.6106 & 548.6106 \\
\hline Organism & 0 & 1155.4586 & 1155.4586 & 0 & 0 & 0 & 0 & 1155.4586 & 555.8557 & 0 & 0 & 0 & 0 & 555.8557 & 555.8557 \\
\hline Solslds & 0 & 7562.3115 & 7562.3115 & 0 & 0 & 0 & 0 & 7562.3115 & 6719.6051 & 0 & 0 & 0 & 0 & 8383.7513 & 8383.7513 \\
\hline Gypsum & 0 & 21.1142 & 21.1142 & 0 & 0 & 0 & 0 & 21.1142 & 0.4063 & 0 & 0 & 0 & 0 & 0.4063 & 0.4063 \\
\hline Lgnsol & 0 & 753.8397 & 753.8397 & 0 & 0 & 0 & 0 & 753.8397 & 669.8356 & 0 & 0 & 0 & 0 & 669.8354 & 669.8354 \\
\hline HMF & 0.115 & 124.0108 & 124.0108 & 0 & 0 & 0 & 0 & 123.844 & 109.8329 & 0.0888 & 0 & 0 & 0 & 109.8329 & 109.8329 \\
\hline C5Sugar & 0 & 1119.4754 & 1119.4754 & 0 & 0 & 0 & 0 & 1119.4754 & 994.7265 & 0 & 0 & 0 & 0 & 994.7264 & 994.7264 \\
\hline C6Sugar & 0 & 2966.165 & 2966.165 & 0 & 0 & 0 & 0 & 2966.165 & 2568.4759 & 0 & 0 & 0 & 0 & 2568.4759 & 2568.4759 \\
\hline C5Solid & 0 & 222.7605 & 222.7605 & 0 & 0 & 0 & 0 & 222.7605 & 4.2865 & 0 & 0 & 0 & 0 & 551.1934 & 551.1934 \\
\hline C6Solid & 0 & 56.697 & 56.697 & 0 & 0 & 0 & 0 & 56.697 & 1.091 & 0 & 0 & 0 & 0 & 655.3348 & 655.3348 \\
\hline $\mathrm{CaH} 2 \mathrm{O} 2$ & 0 & 2.5726 & 2.5726 & 0 & 0 & 0 & 0 & 2.5726 & 0 & 0 & 0 & 0 & 0 & 0 & 0 \\
\hline Ash & 0 & 3391.0043 & 3391.0043 & 0 & 0 & 0 & 0 & 3391.0043 & 65.2523 & 0 & 0 & 0 & 0 & 1043.2097 & 1043.2097 \\
\hline Ethanol & 45.0873 & 151.7402 & 151.743 & 4716.0304 & 18982.6157 & 4716.0304 & 4716.0304 & 31.1905 & 0.3324 & 14.7626 & 0 & 0 & 0 & 0.3947 & 0.3947 \\
\hline Water & 55096.5895 & 265991.385 & 265991.385 & 2024.1179 & 107.9716 & 2024.1179 & 2024.1179 & 198777.387 & 63064.5348 & 29094.0773 & 28809.6111 & 28809.6111 & 28809.6093 & 66617.9136 & 66617.9136 \\
\hline Furfural & 61.7581 & 213.5954 & 213.5969 & 0 & 0.0591 & 0 & 0 & 70.2409 & 3.1367 & 236.1192 & 0 & 0 & 0 & 3.1367 & 3.1367 \\
\hline Sulfuric Acid & 0 & 115.139 & 115.139 & 0 & 0 & 0 & 0 & 115.139 & 102.3085 & 0 & 0 & 0 & 0 & 102.3085 & 102.3085 \\
\hline Nitrogen & 0 & 0 & 0 & 0 & 0 & 0 & 0 & 0 & 0 & 0 & 0 & 0 & 0 & 0.0016 & 0.0016 \\
\hline Carbon Dioxide & 0 & 0 & 0 & 0 & 1.1096 & 0 & 0 & 0 & 0 & 0 & 0 & 0 & 0 & 877.2455 & 877.2455 \\
\hline Oxygen & 0 & 0 & 0 & 0 & 0 & 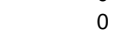 & 0 & 0 & 0 & 0 & 0 & 0 & 0 & 0.0009 & 0.0009 \\
\hline Ammonia & 0 & 0 & 0 & 0 & 0 & 0 & 0 & 0 & 0 & 0 & 0 & 0 & 0 & 0 & \\
\hline Lactic Acid & 1.733 & 2268.6788 & 2268.679 & 0 & 0 & 0 & 0 & 2266.4819 & 2010.4273 & 1.3676 & 0 & 0 & 0 & 2010.4275 & 2010.4275 \\
\hline Acetic Acid & 537.7159 & 4084.1736 & 4084.1727 & 0 & 0 & 0 & 0 & 3552.2003 & 1924.0465 & 178.6808 & 0 & 0 & 0 & 1924.0454 & 1924.0454 \\
\hline Methane & 0 & 0 & 0 & 0 & 0 & 0 & 0 & 0 & 0 & 0 & 0 & 0 & 0 & 347.2569 & 347.2569 \\
\hline Nitrogen Dioxide & 0 & 0 & 0 & 0 & 0 & 0 & 0 & 0 & 0 & 0 & 0 & 0 & 0 & 0 & 0 \\
\hline Sulfur Dioxide & 0 & 0 & 0 & 0 & 0 & 0 & 0 & 0 & 0 & 0 & 0 & 0 & 0 & 0 & 0 \\
\hline DAP & 0 & 202.4815 & 202.4815 & 0 & 0 & 0 & 0 & 202.4815 & 0 & 0 & 0 & 0 & 0 & 0 & 0 \\
\hline
\end{tabular}




\begin{tabular}{|c|c|c|c|c|c|c|c|c|c|c|c|c|c|c|c|}
\hline Stream No. & 8040 & 8042 & 8043 & 8044 & 8045 & 8047 & 8049 & 8597 & 8692 & 8812 & 8813 & 8815 & 8816 & 8818 & 8819 \\
\hline Stream Name & FLUE GAS & \multicolumn{14}{|c|}{ Mol Sieve } \\
\hline $\begin{array}{l}\text { Temp C } \\
\text { Pres bar }\end{array}$ & $\begin{array}{r}165.3185 \\
0.9686\end{array}$ & $\begin{array}{r}25.0489 \\
1.0234\end{array}$ & $\begin{array}{r}220 \\
1\end{array}$ & $\begin{array}{r}48.7963 \\
1\end{array}$ & $\begin{array}{r}820 \\
0.9686\end{array}$ & $\begin{array}{r}639.5961 \\
0.9686\end{array}$ & $\begin{array}{r}270 \\
0.9686\end{array}$ & $\begin{array}{l}147.74 \\
4.4786\end{array}$ & $\begin{array}{r}120.6474 \\
2.0265\end{array}$ & $\begin{array}{r}267.9948 \\
13.1722\end{array}$ & $\begin{array}{r}177 \\
98.9236\end{array}$ & $\begin{array}{r}307.9216 \\
98.2346\end{array}$ & $\begin{array}{r}301.4922 \\
87.7474\end{array}$ & $\begin{array}{r}299.7772 \\
87.7474\end{array}$ & $\begin{array}{r}299.7772 \\
87.7474\end{array}$ \\
\hline Enth MW & -429.03 & -0.01048 & 10.351 & -326.68 & -349.28 & -372.63 & -417.2 & -2.1204 & -6.2795 & -448.67 & -541.4 & -518.81 & -470.21 & -520.46 & -504.85 \\
\hline Vapor mass fraction & 1 & 1 & 1 & 0.76943 & 1 & 1 & 1 & 0 & 0 & 1 & 0 & 0 & 0.99969 & 0 & 0 \\
\hline $\begin{array}{l}\text { Total kgh } \\
\text { Fowrates in } \mathrm{kg} / \mathrm{h}\end{array}$ & 317001.079 & 205000 & 213776.27 & 316989.512 & 317001.136 & 317001.079 & 317001.079 & 497 & 1460.8174 & 124160 & 128000 & 128000 & 128000 & 128000 & 124160 \\
\hline Glucose & 0 & 0 & 0 & 56.8544 & 0 & 0 & 0 & 0 & 0 & 0 & 0 & 0 & 0 & 0 & 0 \\
\hline Cellulose & 0 & 0 & 0 & 7015.2184 & 0 & 0 & 0 & 0 & 0 & 0 & 0 & 0 & 0 & 0 & 0 \\
\hline Xylose & 0 & 0 & 0 & 648.1553 & 0 & 0 & 0 & 0 & 0 & 0 & 0 & 0 & 0 & 0 & 0 \\
\hline Xylan & 0 & 0 & 0 & 3949.0553 & 0 & 0 & 0 & 0 & 0 & 0 & 0 & 0 & 0 & 0 & 0 \\
\hline Lignin & 0 & 0 & 0 & 3579.966 & 0 & 0 & 0 & 0 & 0 & 0 & 0 & 0 & 0 & 0 & 0 \\
\hline Acetate & 0 & 0 & 0 & 548.6106 & 0 & 0 & 0 & 0 & 0 & 0 & 0 & 0 & 0 & 0 & 0 \\
\hline Organism & 0 & 0 & 0 & 555.8557 & 0 & 0 & 0 & 0 & 0 & 0 & 0 & 0 & 0 & 0 & 0 \\
\hline Solslds & 0 & 0 & 0 & 8383.7504 & 0 & 0 & 0 & 0 & 0 & 0 & 0 & 0 & 0 & 0 & 0 \\
\hline Gypsum & 0.4063 & 0 & 0 & 0.4063 & 0.4063 & 0.4063 & 0.4063 & 0 & 0 & 0 & 0 & 0 & 0 & 0 & 0 \\
\hline Lgnsol & 0 & 0 & 0 & 669.8354 & 0 & 0 & 0 & 0 & 0 & 0 & 0 & 0 & 0 & 0 & 0 \\
\hline HMF & 0 & 0 & 0.0001 & 109.833 & 0 & 0 & 0 & 0 & 0 & 0 & 0 & 0 & 0 & 0 & 0 \\
\hline C5Sugar & 0 & 0 & 0 & 994.7263 & 0 & 0 & 0 & 0 & 0 & 0 & 0 & 0 & 0 & 0 & 0 \\
\hline C6Sugar & 0 & 0 & 0 & 2568.4757 & 0 & 0 & 0 & 0 & 0 & 0 & 0 & 0 & 0 & 0 & 0 \\
\hline C5Solid & 0 & 0 & 0 & 551.1934 & 0 & 0 & 0 & 0 & 0 & 0 & 0 & 0 & 0 & 0 & 0 \\
\hline C6Solid & 0 & 0 & 0 & 655.3348 & 0 & 0 & 0 & 0 & 0 & 0 & 0 & 0 & 0 & 0 & 0 \\
\hline $\mathrm{CaH} 2 \mathrm{O} 2$ & 0 & 0 & 0 & 0 & 0 & 0 & 0 & 0 & 0 & 0 & 0 & 0 & 0 & 0 & 0 \\
\hline Ash & 1043.2097 & 0 & 0 & 1043.2097 & 1043.2097 & 1043.2097 & 1043.2097 & 0 & 0 & 0 & 0 & 0 & 0 & 0 & 0 \\
\hline Ethanol & 0 & 0 & 1.1023 & 1.497 & 0 & 0 & 0 & 0 & 0 & 0 & 0 & 0 & 0 & 0 & 0 \\
\hline Water & 83693 & 0 & 391.1629 & 67009.0732 & 83693 & 83693 & 83693 & 497 & 1460.8174 & 124160 & 128000 & 128000 & 128000 & 128000 & 124160 \\
\hline Furfural & 0 & 0 & 4.1699 & 7.3065 & 0 & 0 & 0 & 0 & 0 & 0 & 0 & 0 & 0 & 0 & 0 \\
\hline Sulfuric Acid & 102.3084 & 0 & 0 & 102.3085 & 102.3085 & 102.3084 & 102.3084 & 0 & 0 & 0 & 0 & 0 & 0 & 0 & 0 \\
\hline Nitrogen & 168969.777 & 161950 & 168469 & 168469 & 168969.792 & 168969.777 & 168969.777 & 0 & 0 & 0 & 0 & 0 & 0 & 0 & 0 \\
\hline Carbon Dioxide & 51734.942 & 0 & 17.9352 & 895.1807 & 51735 & 51734.942 & 51734.942 & 0 & 0 & 0 & 0 & 0 & 0 & 0 & 0 \\
\hline Oxygen & 11430.4258 & 43050.0018 & 44890.871 & 44890.8745 & 11430.4329 & 11430.4258 & 11430.4258 & 0 & 0 & 0 & 0 & 0 & 0 & 0 & 0 \\
\hline Ammonia & 0 & 0 & 0 & 0 & 0 & 0 & 0 & 0 & 0 & 0 & 0 & 0 & 0 & 0 & 0 \\
\hline Lactic Acid & 0 & 0 & 0.0009 & 2010.4286 & 0 & 0 & 0 & 0 & 0 & 0 & 0 & 0 & 0 & 0 & 0 \\
\hline Acetic Acid & 0 & 0 & 2.0409 & 1926.0861 & 0 & 0 & 0 & 0 & 0 & 0 & 0 & 0 & 0 & 0 & 0 \\
\hline Methane & 0 & 0 & 0 & 347.2569 & 0 & 0 & 0 & 0 & 0 & 0 & 0 & 0 & 0 & 0 & 0 \\
\hline Nitrogen Dioxide & 0 & 0 & 0 & 0 & 0 & 0 & 0 & 0 & 0 & 0 & 0 & 0 & 0 & 0 & 0 \\
\hline Sulfur Dioxide & 0 & 0 & 0 & 0 & 0 & 0 & 0 & 0 & 0 & 0 & 0 & 0 & 0 & 0 & 0 \\
\hline DAP & 0 & 0 & 0 & 0 & 0 & 0 & 0 & 0 & 0 & 0 & 0 & 0 & 0 & 0 & 0 \\
\hline 2-2-4-Trimth-C5 & 27.0875 & 0 & 0 & 0 & 27.0875 & 27.0875 & 27.0875 & 0 & 0 & 0 & 0 & 0 & 0 & 0 & 0 \\
\hline
\end{tabular}




\begin{tabular}{|c|c|c|c|c|c|c|}
\hline Stream No. & 8821 & 8823 & 8830 & 8872 & 8873 & 9611 \\
\hline Stream Name & \multicolumn{3}{|c|}{ BLR BLOWDOWN } & \multicolumn{3}{|l|}{ TO U1100 } \\
\hline Temp C & 321 & 267.9948 & 267.9948 & 137 & 137 & 179.8844 \\
\hline Pres bar & 113.484 & 13.1722 & 13.1722 & 3.3437 & 3.3437 & 10 \\
\hline Enth MW & -16.67 & -36.136 & -307.58 & -123.28 & -547.74 & -111.95 \\
\hline Vapor mass fraction & 1 & 1 & 1 & 0 & 0 & 0.60031 \\
\hline Total kg/h & 4521 & 10000.001 & 85117.4729 & 28809.9194 & 128000 & 28809.6111 \\
\hline \multicolumn{7}{|l|}{ Fowrates in $\mathrm{kg} / \mathrm{h}$} \\
\hline Glucose & 0 & 0 & 0 & 0 & 0 & 0 \\
\hline Cellulose & 0 & 0 & 0 & 0 & 0 & 0 \\
\hline Xylose & 0 & 0 & 0 & 0 & 0 & 0 \\
\hline Xylan & 0 & 0 & 0 & 0 & 0 & 0 \\
\hline Lignin & 0 & 0 & 0 & 0 & 0 & 0 \\
\hline Acetate & 0 & 0 & 0 & 0 & 0 & 0 \\
\hline Organism & 0 & 0 & 0 & 0 & 0 & 0 \\
\hline Solslds & 0 & 0 & 0 & 0 & 0 & 0 \\
\hline Gypsum & 0 & 0 & 0 & 0 & 0 & 0 \\
\hline Lgnsol & 0 & 0 & 0 & 0 & 0 & 0 \\
\hline HMF & 0 & 0 & 0 & 0 & 0 & 0 \\
\hline C5Sugar & 0 & 0 & 0 & 0 & 0 & 0 \\
\hline C6Sugar & 0 & 0 & 0 & 0 & 0 & 0 \\
\hline C5Solid & 0 & 0 & 0 & 0 & 0 & 0 \\
\hline C6Solid & 0 & 0 & 0 & 0 & 0 & 0 \\
\hline $\mathrm{CaH} 2 \mathrm{O} 2$ & 0 & 0 & 0 & 0 & 0 & 0 \\
\hline Ash & 0 & 0 & 0 & 0 & 0 & 0 \\
\hline Ethanol & 0 & 0 & 0 & 0 & 0 & 0 \\
\hline Water & 4521 & 10000.001 & 85117.4729 & 28809.9194 & 128000 & 28809.6111 \\
\hline Furfural & 0 & 0 & 0 & 0 & 0 & 0 \\
\hline Sulfuric Acid & 0 & 0 & 0 & 0 & 0 & 0 \\
\hline Nitrogen & 0 & 0 & 0 & 0 & 0 & 0 \\
\hline Carbon Dioxide & 0 & 0 & 0 & 0 & 0 & 0 \\
\hline Oxygen & 0 & 0 & 0 & 0 & 0 & 0 \\
\hline Ammonia & 0 & 0 & 0 & 0 & 0 & 0 \\
\hline Lactic Acid & 0 & 0 & 0 & 0 & 0 & 0 \\
\hline Acetic Acid & 0 & 0 & 0 & 0 & 0 & 0 \\
\hline
\end{tabular}




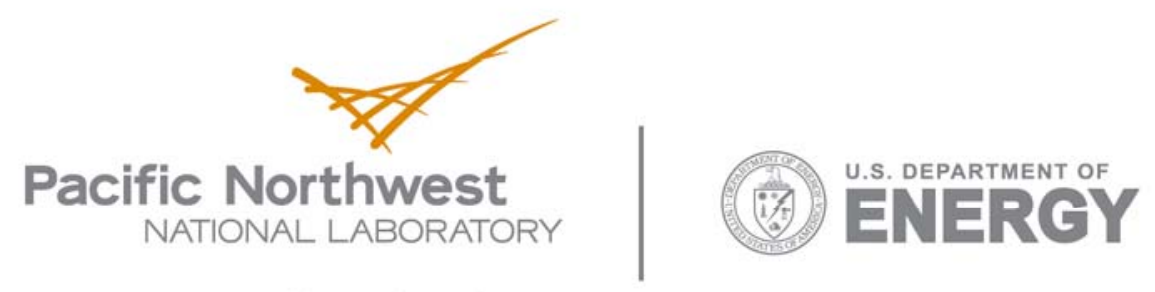

902 Battelle Boulevard

P.O. Box 999

Richland, WA 99352

1-888-375-PNNL (7665)

www.pnl.gov 